\title{
Wave and vortex dynamics on the surface of a sphere
}

\author{
By LORENZO M. POLVANI ${ }^{1}$ AND DAVID G. DRITSCHEL ${ }^{2}$ \\ ${ }^{1}$ Department of Applied Physics, Columbia University, New York, NY 10027, USA \\ ${ }^{2}$ Department of Applied Mathematics and Theoretical Physics, University of Cambridge, \\ Silver Street, Cambridge CB3 9EW, UK
}

(Received 5 March 1992 and in revised form 22 March 1993)

Motivated by the observed potential vorticity structure of the stratospheric polar vortex, we study the dynamics of linear and nonlinear waves on a zonal vorticity interface in a two-dimensional barotropic flow on the surface of a sphere (interfacial Rossby waves). After reviewing the linear problem, we determine, with the help of an iterative scheme, the shapes of steadily propagating nonlinear waves; a stability analysis reveals that they are (nonlinearly) stable up to very large amplitude.

We also consider multi-vortex equilibria on a sphere: we extend the results of Thompson (1883) and show that a (latitudinal) ring of point vortices is more unstable on the sphere than in the plane; notably, no more than three point vortices on the equator can be stable. We also determine the shapes of finite-area multi-vortex equilibria, and reveal additional modes of instability feeding off shape deformations which ultimately result in the complex merger of some or all of the vortices.

We discuss two specific applications to geophysical flows: for conditions similar to those of the wintertime terrestrial stratosphere, we show that perturbations to a polar vortex with azimuthal wavenumber 3 are close to being stationary, and hence are likely to be resonant with the tropospheric wave forcing; this is often observed in highresolution numerical simulations as well as in the ozone data. Secondly, we show that the linear dispersion relation for interfacial Rossby waves yields a good fit to the phase velocity of the waves observed on Saturn's 'ribbon'.

\section{Introduction}

The dynamics of the stratospheric polar vortex has attracted much research interest in recent years in the light of mounting evidence of man's adverse impact on the environment (e.g. the ozone hole-see McIntyre 1991 for a review). With few exceptions, however, most of the work to date has been concentrated on the development of complex two- and three-dimensional numerical models. These giant models have somehow obscured the fact that a number of basic dynamical questions can be addressed in a simpler context.

With this in mind, we consider in this paper the vorticity dynamics of a twodimensional sheet of constant-density ideal fluid on the surface of a sphere (commonly referred to as the barotropic model), possibly the simplest system by which atmospheric flows may be approximated. Far from being a useless oversimplification, the barotropic model has already provided rich insights into the evolution of the polar vortex (Juckes \& McIntyre 1987; Polvani \& Plumb 1993; Waugh 1992). In this paper, we concentrate on the dynamics of waves and vortices in a barotropic spherical fluid; in particular, we 


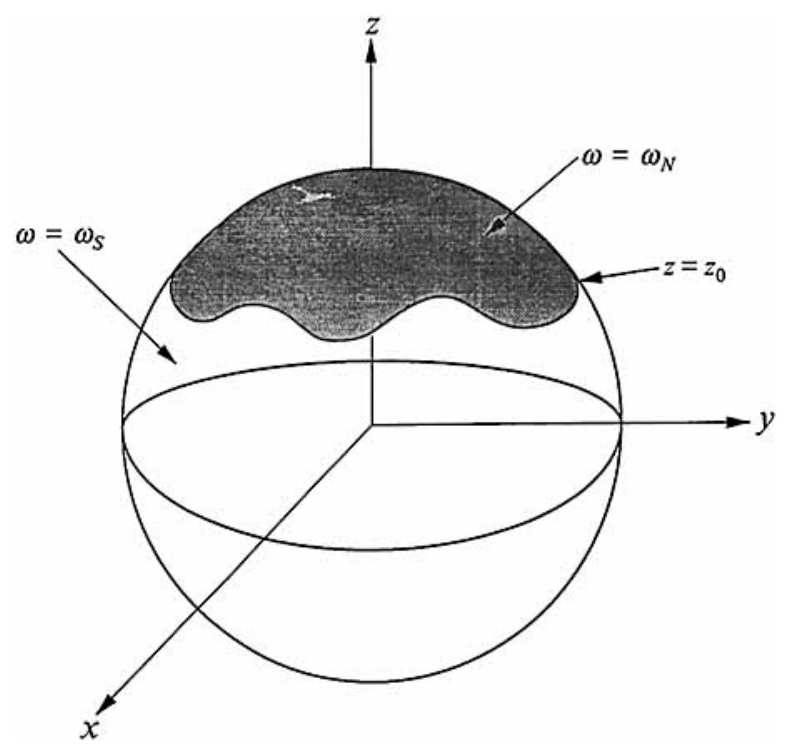

FIGURE 1. A sketch of the vorticity distribution for a one-contour polar cap. The unperturbed edge of the polar cap is at $\vartheta=\vartheta_{0}$, i.e. $z(\equiv \sin \vartheta)=z_{0}$.

study the question of the existence and stability of nonlinear wave and vortex equilibria on the surface of a sphere.

For a barotropic atmosphere in solid-body rotation, the vorticity is zonally symmetric and proportional to the sine of latitude, i.e. the axial Cartesian coordinate. However, it is by now well established that the potential vorticity distribution in the wintertime stratosphere is very far from solid-body rotation; on isentropic surfaces one finds a latitudinal band of very steep potential vorticity gradients separating two regions of rather weak gradients, the 'inside' of the polar vortex to the north and the 'outside' to the south. Numerical studies have shown (Juckes \& McIntyre 1987) that it is the dynamical effect of breaking Rossby waves, induced by the planetary wave forcing from the troposphere, that leads to the formation of such a steep gradient separating two regions of relatively uniform isentropic potential vorticity. This phenomenon, named vortex stripping, has recently been the object of an extensive study (Dritschel \& Legras 1993).

In view of this, it would seem reasonable to start by considering the simplest possible (relevant) vorticity distribution for a two-dimensional ideal fluid on the surface of a sphere, namely a single zonal vorticity jump separating the spherical surface into two regions of uniform vorticity, as shown in figure 1 . We start by considering the simplest case where the vorticity interface is zonally symmetric (i.e. a latitudinal circle), for which the flow field is easily calculated. We then consider perturbations to this zonal boundary and obtain the linear dispersion relation for waves on the vorticity interface. These waves are in fact Rossby waves, since the restoring mechanism responsible for wave propagation is provided by the presence of a vorticity gradient; we call them interfacial Rossby waves. The next step consists in considering waves of finite amplitude, and determining those shapes that propagate without deformation.

Such finite-amplitude steadily propagating vorticity waves were first determined for perturbations to a circular vortex on a plane by Deem \& Zabusky (1978), who coined the name 'V-state' (i.e. vortex state). Since then, similar equilibria have been found for a variety of planar vorticity distributions: dipolar (Pierrehumbert 1980), tripolar 
(Polvani \& Carton 1990), multipolar (Dritschel 1985), for vortex streets (Saffman \& Schatzman 1982), as well as for multi-contour (Dritschel 1989) and multi-layer systems (Polvani 1989, 1991). To the best of our knowledge, the results presented here are the first known nonlinear wave and vortex equilibria obtained for spherical geometry.

In $\S 2$, waves on a zonal vorticity interface are considered. The problem is set up in $\S 2.1$, and the linear results are reviewed in $\$ 2.2$. The algorithm used to obtain the nonlinear wave solutions is presented in $\$ 2.3$. Ironically, the method employed relies heavily on the use of Cartesian coordinates instead of spherical ones since, as we will show, this choice considerably simplifies the calculation. The solutions for largeamplitude nonlinear waves propagating without change in shape on a vorticity interface are presented and discussed in $\$ 2.4$. Finally, the stability of these waves is considered in $\S 2.5$. Surprisingly, we find that most of these waves are stable to finiteamplitude perturbations even when the flow departs very greatly from zonal symmetry.

In $\S 3$ we consider corotating multiple-vortex equilibria. From a physical viewpoint, such solutions are relevant to major warming events in the stratosphere, when the polar vortex is broken up into a number of pieces (the breaking into two smaller vortices has been repeatedly observed). From a mathematical viewpoint, these solutions are of interest because of their connection to known results in planar geometry. For instance, it is well known that a system of $N$ point vortices, placed along a circle is unstable for $N \geqslant 8$ (Thompson 1883), but finite-area vortices can be unstable even for $N=2$ (Dritschel 1985). We have extended these results to the sphere, for both the point and finite-area multiple-vortex equilibria, in $\$ 3.1$ and $\$ 3.2$ respectively.

The results summarized in $\S 4$, where two specific applications are discussed: the first to the dynamics of the stratospheric polar vortex, the second to the waves on Saturn's 'ribbon'.

\section{Linear and nonlinear interfacial Rossby waves}

\subsection{The basic flow}

We consider the dynamics of an inviscid, incompressible, unforced and infinitesimally thin fluid shell on the surface of a sphere of unit radius. We will be working throughout this paper (except where noted) in a frame of reference that is fixed in space, as opposed to the more common meterological convention of choosing a frame rotating with the Earth. The barotropic analogue of the quantity of dynamical importance (the isentropic potential vorticity) is what is usually referred to as the 'absolute vorticity', which is equal to the vorticity in the rotating frame of the Earth plus the Coriolis term $f=2 \Omega_{e} \sin \vartheta\left(\Omega_{e}\right.$ being the Earth's angular velocity and $\vartheta$ the latitude).

Since the absolute vorticity is the quantity that is nearly piecewise uniform in the flows that we are interested in, it is more convenient to move to an absolute frame and simply consider the vorticity in that frame, which is precisely the absolute vorticity. The reader should not be confused as to whether the dynamics described here are those of a rotating fluid or not; a simple change of frame cannot possibly affect the dynamics. What matters is what vorticity distribution is relevant to the flow one is considering. The stratospheric polar vortex is characterized by near-uniform regions of absolute vorticity and therefore using an absolute frame leads to much simplification.

In that frame, the barotopic dynamics is dictated by the material conservation of vorticity,

$$
\left(\frac{\partial}{\partial t}+\frac{u}{\cos \vartheta} \frac{\partial}{\partial \varphi}+v \frac{\partial}{\partial \vartheta}\right) \omega=0
$$


where $\vartheta$ is the latitude, $\varphi$ the longitude, and $u$ and $v$ are the zonal and meridional velocities. (All quantities and expressions in this paper are non-dimensional, except where otherwise noted.) The vorticity $\omega$ is the two-dimensional spherical Laplacian of the stream function $\psi$, namely

$$
\omega=\nabla^{2} \psi=\frac{1}{\cos \vartheta} \frac{\partial}{\partial \vartheta}\left(\cos \vartheta \frac{\partial \psi}{\partial \vartheta}\right)+\frac{1}{\cos ^{2} \vartheta} \frac{\partial^{2} \psi}{\partial \varphi^{2}},
$$

and the velocities are related to the stream function via the geostrophic relations:

$$
u=-\frac{\partial \psi}{\partial \vartheta} \quad \text { and } \quad v=\frac{1}{\cos \vartheta} \frac{\partial \psi}{\partial \varphi} .
$$

Note that the latitude $\vartheta$ is not the correct conjugate variable to the longitude $\varphi$. The proper choice is $z \equiv \sin \vartheta$, the axial component in Cartesian coordinates, from which Hamilton's equations are recovered, namely

$$
\frac{\mathrm{d} \varphi}{\mathrm{d} t}=-\frac{\partial \psi}{\partial z} \text { and } \frac{\mathrm{d} z}{\mathrm{~d} t}=\frac{\partial \psi}{\partial \varphi} .
$$

Notice also that an area element on the spherical surface takes the simple form $\mathrm{d} z \mathrm{~d} \varphi$. We have found, in general, that the use of Cartesian coordinates leads to major simplifications to all expressions. For instance, the three Cartesian components of the velocity vector are obtained from the stream function from the simple expression

$$
\mathrm{d} \boldsymbol{x} / \mathrm{d} t=\boldsymbol{x} \times \boldsymbol{\nabla} \psi
$$

Consider now the piecewise-uniform vorticity distribution for an unperturbed (i.e. zonally symmetric) polar cap, namely $\omega=\omega_{N}$ north of some latitude $\vartheta_{0}$, and $\omega=\omega_{S}$ to the south. The flow associated with this vorticity field is obviously zonal (i.e. $v=0$ ) and, may be obtained by integrating (2), and substituting into (3). However, it is easier to work in Cartesian coordinates. Thus, rewriting (2) as

$$
-\frac{\mathrm{d}}{\mathrm{d} z}\left[\left(1-z^{2}\right)^{\frac{1}{2}} u\right]=\omega
$$

yields immediately

$$
u=\left\{\begin{aligned}
\omega_{N}[(1-z) /(1+z)]^{\frac{1}{2}} & \text { for } \quad z>z_{0} \\
-\omega_{S}[(1+z) /(1-z)]^{\frac{1}{2}} & \text { for } \quad z<z_{0} .
\end{aligned}\right.
$$

Note that continuity of the velocity field at the edge of the polar cap $\left(z=z_{0} \equiv \sin \vartheta_{0}\right)$, leads to the constraint

$$
\left(1-z_{0}\right) \omega_{N}+\left(z_{0}+1\right) \omega_{S}=0 .
$$

Hence $z_{0}, \omega_{N}$ and $\omega_{S}$ cannot be specified independently. This is due to the well-known fact that the integral of the vorticity over the whole spherical surface (the left-hand side of (6)) must be identically zero. The flow given by (5), a simple zonal jet peaking at the edge of the polar vortex, $\uparrow$ is an exact time-independent solution of (1). We next consider the stability of this flow.

$\uparrow$ To obtain the flow in a frame of reference rotating with an angular velocity $\frac{1}{2} f$ (the conventional frame in metrology), it is sufficient to subtract a term $\left(\frac{1}{2} f\right) \cos \vartheta=\left(\frac{1}{2} f\right)\left(1-z^{2}\right)^{\frac{1}{2}}$ from the velocities in (5). 


\subsection{The linear waves}

Since the vorticity gradient never changes sign with latitude, one expects that the flow (5) is linearly stable under small perturbations (cf. Rayleigh's theorem); in fact it is stable to finite-amplitude perturbations (Dritschel 1988a). Hence the question of interest is to determine the angular velocity of normal mode perturbations on the vorticity interface at $z=z_{0}$. Instead of proceeding by solving for the perturbed flow in both regions and matching at the boundary, is it much simpler to deal with the position of the vorticity jump alone. This type of analysis has been carried out previously (Dritschel 1988b, Appendix A; and Dritschel \& Polvani 1992) and we briefly summarize it here for clarity and completeness.

Let the perturbed position of the vorticity jump be

$$
z=z_{\mathbf{0}}+z^{\prime}(\varphi, t),
$$

where $z_{0}$ is the undisturbed position and $z^{\prime}$ the perturbation (see figure 1 ). The stability problem then reduces to writing a linear evolution equation for $z^{\prime}(\varphi, t)$. This is easily obtained by using the vertical component of the equations of motion (Dritschel 1988c)

$$
\frac{\mathrm{d} x}{\mathrm{~d} t}=\boldsymbol{u}(\boldsymbol{x})=-\frac{1}{4 \pi} \sum_{k} \tilde{\omega}_{k} \oint_{\mathscr{C}_{k}} \log \left|\boldsymbol{x}-\boldsymbol{X}_{k}\right|^{2} \mathrm{~d} X_{k},
$$

which gives the velocity field at any point on the sphere from a given vorticity distribution that is piecewise uniform, with vorticity jumping by the values $\tilde{\omega}_{k}$ across the contours $\mathscr{C}_{k}$ (for a rederivation of (7) see Dritschel \& Polvani 1992, Appendix A). For the one-contour polar-cap vorticity distribution shown in figure 1 , the $z$ component of (7) gives

$$
\frac{\mathrm{d} z^{\prime}}{\mathrm{d} t}=\frac{\partial z^{\prime}}{\partial t}+\Omega_{0} \frac{\partial z^{\prime}}{\partial \varphi}=-\frac{\tilde{\omega}}{4 \pi} \int_{0}^{2 \pi} \log |x(\alpha)-x(\varphi)|^{2} \frac{\partial z^{\prime}}{\partial \alpha} \mathrm{d} \alpha,
$$

where $\tilde{\omega}=\omega_{N}-\omega_{S}$ is the vorticity jump, and $\Omega_{0}=\frac{1}{2} \tilde{\omega}$ is the angular velocity of the undisturbed flow. The dispersion relation is obtained by letting

$$
z^{\prime}(\varphi, t)=\hat{z} \mathrm{e}^{\mathrm{i}(m \varphi-\sigma t)}+\text { c.c. }
$$

where $m$ is the azimuthal wavenumber, $\sigma$ the frequency and $\hat{z}$ a constant amplitude. Upon substitution into (8), using the undisturbed quantities inside the logarithmic expression and the identity

$$
\frac{1}{2 \pi} \int_{0}^{2 \pi} \frac{\sin \beta \sin (m \beta)}{1-\cos \beta} \mathrm{d} \beta=1 \quad \text { for all } m,
$$

one obtains the very simple dispersion relation

$$
\Omega=\Omega_{0}-\tilde{\omega} / 2 m,
$$

where $\Omega \equiv \sigma / m$. It is perhaps surprising that this expression is identical to the one for a planar circular vortex (see Lamb 1932, §158) - it is independent of the latitude $\vartheta_{0}$ of the undisturbed polar cap. The first term is of course a simple Doppler shift, due to the advection of the perturbations by the mean flow. The second term, representing the angular velocity of the perturbations in a frame that moves with the mean flow, is always opposite in sign to the vorticity jump $\tilde{\omega}$ (this is of course the characteristic signature of 'westward'-propagating Rossby waves). It is also interesting to note that, 


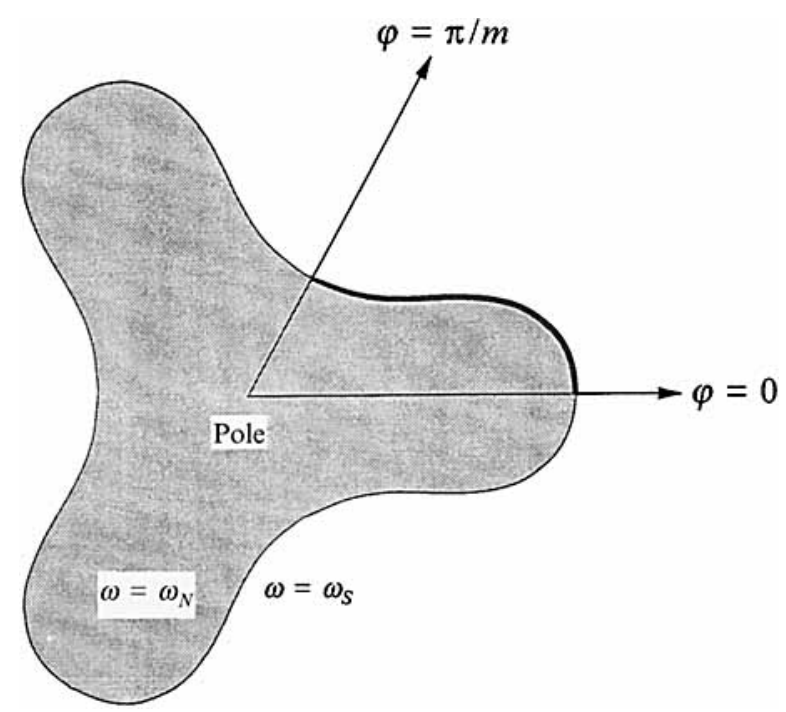

FIGURE 2. A sketch of the nonlinear solutions as seen from the north pole. Because of symmetry, only the portion $0<\varphi<(\pi / m)$ needs to be computed. At large amplitude, the contour is not a singlevalued function of the longitude $\varphi$.

in the long-wavelength limit $\Omega$ varies as $1 / m$ and not $1 / m^{2}$ as is the case for the traditional Rossby waves. An application of this to a feature on Saturn is discussed in $\S 4$.

\subsection{The algorithm for the nonlinear problem}

We now abandon the restrictions of linear theory, and address the question of largeamplitude interfacial spherical Rossby waves. In particular, we wish to determine those perturbations of the polar cap that rotate at a fixed angular velocity without change in shape. Both the angular velocity and the shape are unknowns that have to be determined by solving a nonlinear problem. In this section we discuss the main features of the numerical algorithm that we have used to obtain the equilibrium vorticity distributions. The actual results, including the nonlinear dispersion relations and shapes as well as questions of stability, are presented in the next two subsections. $\dagger$

The basic set-up is sketched in figure 2 . Given a region of uniform vorticity $\omega_{N}$, centred around the pole and having $m$-fold symmetry, we have to find those shapes that rotate at constant angular velocity $\Omega$ without deformation. These shapes, which are finite-amplitude extensions of the linear waves discussed in the previous subsection, are then exact nonlinear solutions of (1). Such solutions were originally found for a vortex patch on the plane by Deem \& Zabusky (1978), and we are here extending that problem to spherical geometry. The novelty resides in that, while on an infinite plane there is no characteristic lengthscale, there does exist one on a spherical surface, namely the sphere's radius. This means that, compared to the planar case, an additional parameter is necessary to describe the solutions of the spherical problem, and for intuitive reasons, we take this to be the latitude $\vartheta_{0}$ of the undisturbed vortex.

Apart from the wavenumber $m$, the other parameter needed to identify each solution is then related to the amplitude of the wave. Given the Cartesian coordinates $(x, y, z)$

$\dagger$ Since the discussion of this subsection is of a rather technical nature, we recommend that those mainly interested in the physical results read only the first three paragraphs of this section, where the problem is set up and the basic parameters are defined, and then proceed directly to the next subsection. 
of a point on the sphere, define the variable $\mathscr{Z} \equiv 1-z$. From symmetry considerations, it is clear that only the portion of the vortex boundary with $0<\varphi<(\pi / m)$ need be determined. The solutions are parametrized by the aspect ratio $\lambda$, defined as

$$
\lambda \equiv \frac{\mathscr{Z}(\varphi=0)}{\mathscr{Z}(\varphi=\pi / n)} .
$$

A given solution is then described by a wavenumber $m$, an 'average latitude' of the boundary $\vartheta_{0}$ and an aspect ratio $\lambda$. Linear theory corresponds to $|\lambda-1| \ll 1$, while the planar solutions of Deem \& Zabusky correspond to $\mathscr{Z}(\varphi) \ll 1$.

To determine the shape of the vortex, a parameterization of the boundary is needed. The simplest way to do this would be to use the longitude $\varphi$. This was our original approach, but we soon discovered that, at large amplitude, the vortex boundary becomes multiple-valued in $\varphi$ as the perturbations 'fold-over'. Hence a more flexible formalism is needed. We have used the arclength $s$ along the boundary as the independent variable that parametrizes the boundary of the polar cap, which is then described by the three functions $\boldsymbol{x}=\boldsymbol{x}(s)$, subject to the constraint

$$
|x|=\left(x^{2}+y^{2}+z^{2}\right)^{\frac{1}{2}}=1 .
$$

The algorithm we use is an iteration scheme. From the value of $x(s)$ at the $n$th iteration, which we write $\boldsymbol{x}_{n}(s)$, the algorithm finds $\boldsymbol{x}_{n+1}(s)$ at the next iteration by letting

$$
\boldsymbol{x}_{n+1}(s)=\boldsymbol{x}_{n}(s)+\boldsymbol{x}^{\prime}(s)=\boldsymbol{x}_{n}(s)+\eta(s) \hat{\boldsymbol{n}} .
$$

The small correction $\boldsymbol{x}^{\prime}(s)$ is made in the direction of the normal $\hat{n}=\boldsymbol{x}_{n} \times \mathrm{d} \boldsymbol{x}_{n} / \mathrm{d} s$ to the vortex boundary.

It is then necessary to relate the function $\eta(s)$ to the stream function $\psi$ along the boundary, and this is accomplished as follows. First one transfers to a frame that rotates with the unknown angular velocity $\Omega$. The stream function $\psi_{r}$ in that rotating frame is then given by

$$
\psi_{r}(\boldsymbol{x})=\psi(\boldsymbol{x})-\Omega(1-z)=\psi(\boldsymbol{x})-\boldsymbol{\Omega} \mathscr{Z} .
$$

In the limit $\mathscr{Z} \ll 1$, the second term reduces to $\frac{1}{2} \Omega r^{2}$, so that the familiar planar expression is recovered near the pole, as expected.

In the rotating frame the boundary of the polar cap is, of course, stationary; this implies that the boundary itself is a streamline, $\psi_{r}=C$. Substituting (10) into (11), and performing a straightforward Taylor expansion yields, after truncation to first-order terms (the primed terms),

$$
\begin{aligned}
\psi_{r}\left(\boldsymbol{x}_{n+1}\right) & =\psi\left(\boldsymbol{x}_{n+1}\right)-\Omega_{n+1} \mathscr{Z}_{n+1}=C \\
& \approx \psi\left(\boldsymbol{x}_{n}\right)+\eta(\hat{\boldsymbol{n}} \cdot \boldsymbol{\nabla}) \psi\left(\boldsymbol{x}_{n}\right)-\Omega_{n}\left(\mathscr{Z}_{n}-\eta n_{z}\right)-\Omega^{\prime} \mathscr{Z}_{n} \\
& =\psi_{r}\left(\boldsymbol{x}_{n}\right)+\eta\left[(\hat{\boldsymbol{n}} \cdot \boldsymbol{\nabla}) \psi\left(\boldsymbol{x}_{n}\right)+\Omega_{n} n_{z}\right]-\Omega^{\prime} \mathscr{Z}_{n},
\end{aligned}
$$

where we have defined $\Omega^{\prime} \equiv \Omega_{n+1}-\Omega_{n}$ (recall that $\Omega$ is also an unknown that needs to be iterated). Inverting this, one obtains an expression for $\eta$ in terms of quantities that are known at the $n$th iteration (except $C$ and $\Omega^{\prime}-$ see immediately below):

$$
\eta=\frac{C-\psi_{r}\left(\boldsymbol{x}_{n}\right)+\Omega^{\prime} \mathscr{Z}_{n}}{\chi\left(\boldsymbol{x}_{n}\right)},
$$

where $\chi\left(\boldsymbol{x}_{n}\right) \equiv(\hat{n} \cdot \nabla) \psi\left(\boldsymbol{x}_{n}\right)+\Omega_{n} n_{z}$. The two constants $C$ and $\Omega^{\prime}$ are as yet undetermined. They are chosen in such a way that the area and the aspect ratio of the 


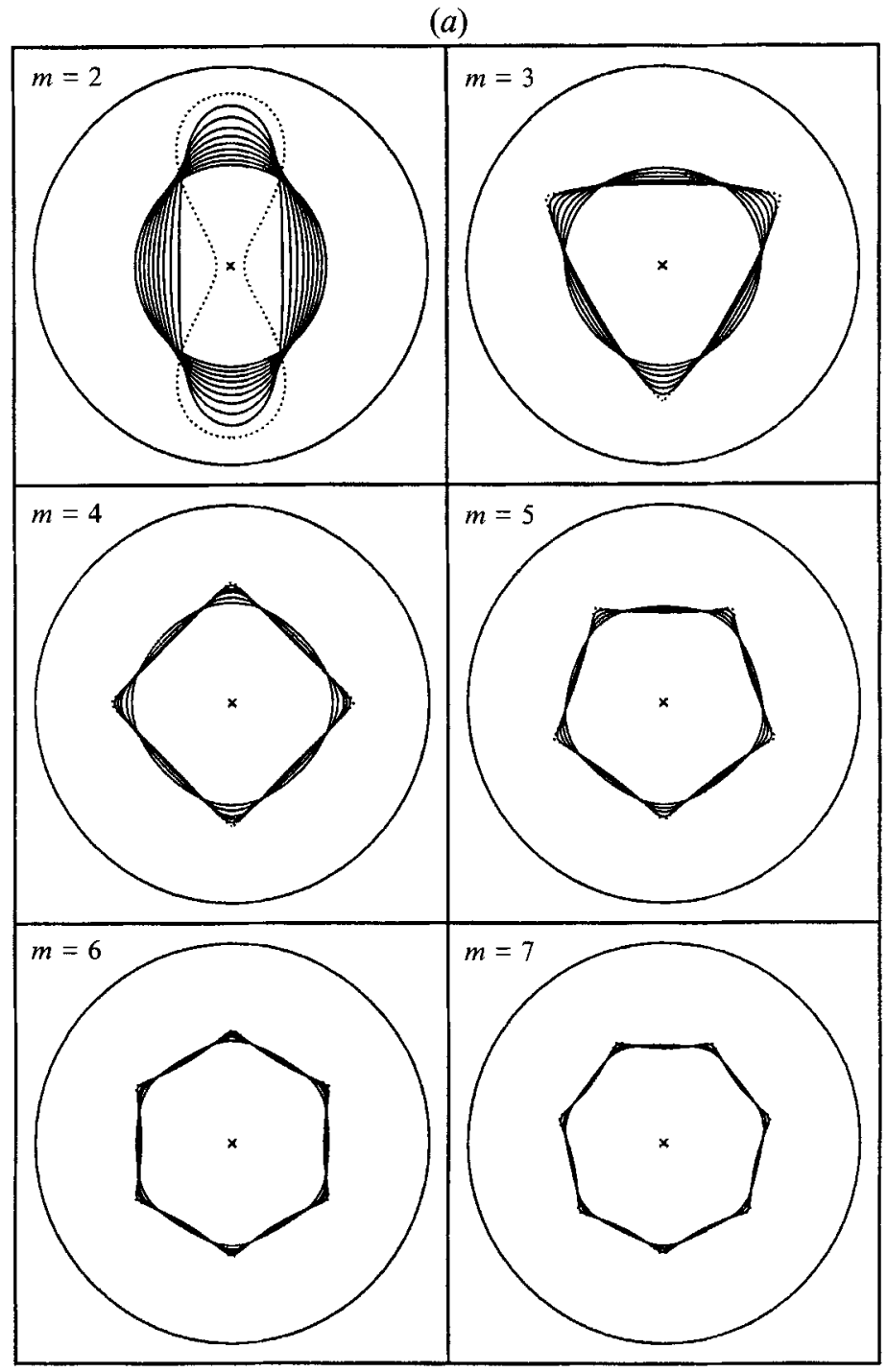

(b)

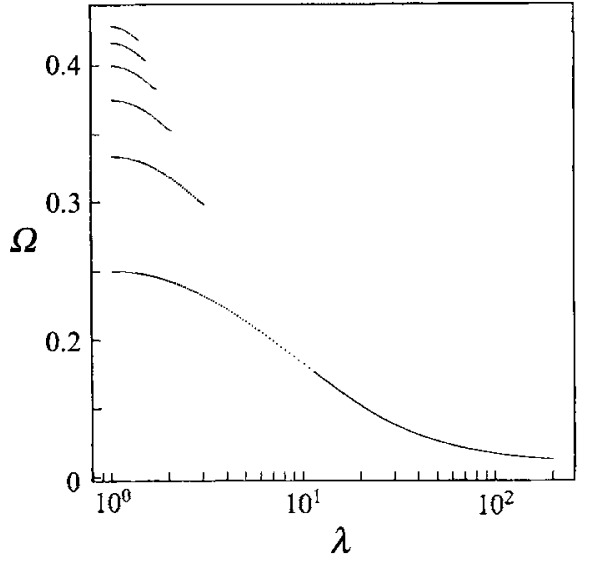


solution be equal to fixed values specified at the beginning of the iteration. These two constraints yield a simple linear system for $C$ and $\Omega^{\prime}$ at each iteration:

$$
a_{1} C+b_{1} \Omega^{\prime}=c_{1}, \quad a_{2} C+b_{2} \Omega^{\prime}=c_{2},
$$

where the $a, b$ and $c$ are six constants that can be evaluated from the known solution at the $n$th iteration (see Appendix A). Solving for $C$ and $\Omega^{\prime}$ in (14), the $(n+1)$ th iterate is then uniquely specified in terms of the $n$th one via (13) and (10).

A first guess for $x(s)$ and $\Omega$ is necessary to start the iteration. In practice we proceed by fixing $z_{0}$ and finding solutions at progressively increasing aspect ratio $\lambda$ for a given wavenumber $m$. For $\lambda \approx 1$ linear theory provides a good guess. As $\lambda$ becomes large, we use the converged solution at a given $\lambda$ as a guess for the solution at slightly larger $\lambda$. We proceed in this way until, at large amplitudes, the algorithm fails to converge. To test convergence, we compute at each iteration the $L_{1}$-norm of the vector $x_{n+1}-x_{n}$, and stop the process when $\left|\boldsymbol{x}_{n+1}-\boldsymbol{x}_{n}\right|<\epsilon$.

This is only the skeleton of the algorithm. More details are given in Appendix A, including the expressions for the constants in (14), and the formulae needed to compute $\chi$ and $\psi$ in terms of contour integrals. All the solutions presented in this paper were obtained with the contour discretization of Contour Surgery (as detailed in Dritschel 1989), which is cubic in the deviations from a straight line. Also, all the contour integrals are evaluated with cubic splines. Hence the algorithm is $O\left(1 / N^{4}\right)$, where $N$ is the number of nodes that discretize the vortex boundary.

\subsection{The nonlinear waves}

We now discuss the nonlinear wave solutions obtained with this algorithm. These waveforms are exact nonlinear solutions of (1). Each solution is characterized by three parameters: a wavenumber $m$, an aspect ratio $\lambda>1$, and a latitude $\vartheta_{0}$ of the edge of the corresponding circular polar cap. The equilibria have area equal to $2 \pi\left(1-\sin \vartheta_{0}\right)$, independent of $m$ and $\lambda$. To reduce the computational task, we have computed only the first six wavenumbers $(m=2, \ldots, 7)$, and restricted our attention to three representative latitudes: $\vartheta_{0}=60^{\circ}, 30^{\circ}, 0^{\circ}$. Needless to say, near the pole $\left(\vartheta_{0}=90^{\circ}\right)$ one recovers the 'V-state' solutions of Deem \& Zabusky (1978). The solutions presented here were obtained with typically 250 nodes on each contour and a convergence criterion of $\epsilon=10^{-6}$, requiring from a handful of iterations for the solutions near the linear regime to a hundred or so iterations for the very large-amplitude waves.

We start with the parameter values more directly of interest to the stratospheric polar vortex, namely $\vartheta_{0}=60^{\circ}$. The solutions for the polar-cap boundary are shown in figure 3(a) (we have computed many more solutions than the ones shown in this figure). The stability of these equilibria will be extensively discussed in the next section; with regard to figure $3(a)$ it suffices to say at this stage that the solid lines represent stable nonlinear equilibrium solutions. The solid line with largest amplitude is the largestamplitude stable equilibrium that was found. The dotted line is the largest-amplitude unstable equilibrium (at larger amplitude our algorithm does not converge). Hence the

FIGURE 3. (a) The nonlinear wave solutions for $\vartheta_{0}=60^{\circ}$. These are polar stereographic views from the north pole, indicated by the cross. The outer circle is the equator. The solid lines represent stable solutions for the edge of the polar cap at progressively increasing amplitudes of the wave. The dotted lines are the largest-amplitude unstable solutions, as described in the text (see $\$ 2.5)$. (b) The nonlinear dispersion relations for the equilibria shown in $(a)$. The values of $\Omega$ are given in units of $\tilde{\omega}$, the vorticity jump across the interface. Recall that the linear dispersion relation is $\Omega=(m-1) /(2 m)$. The dotted part of the line shows the stable solutions, each dot corresponding to a different equilibrium. The solid portions of each line are the unstable solutions. Each line corresponds to a different $m, 2 \leqslant m \leqslant 7$. 


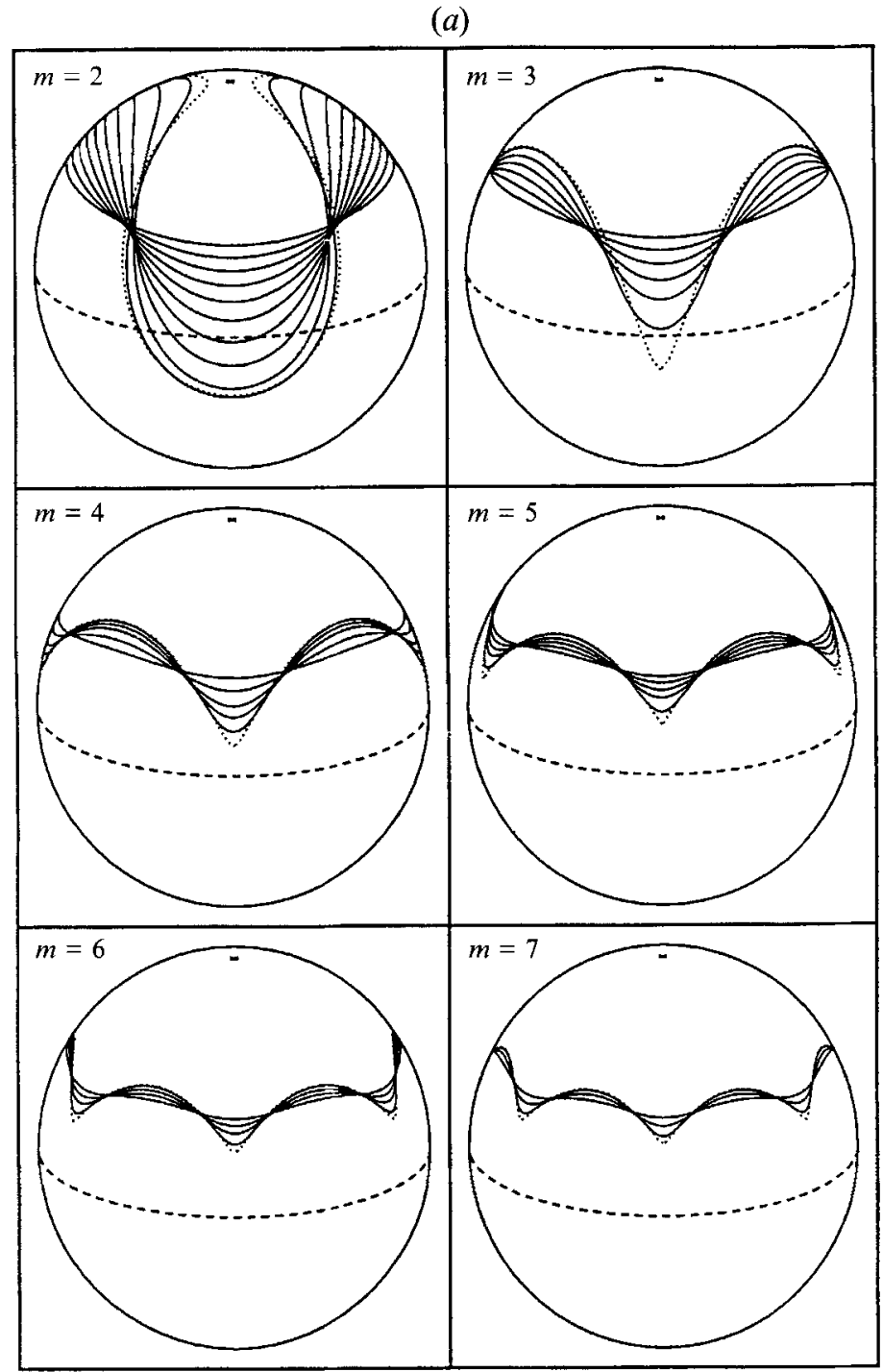

(b)

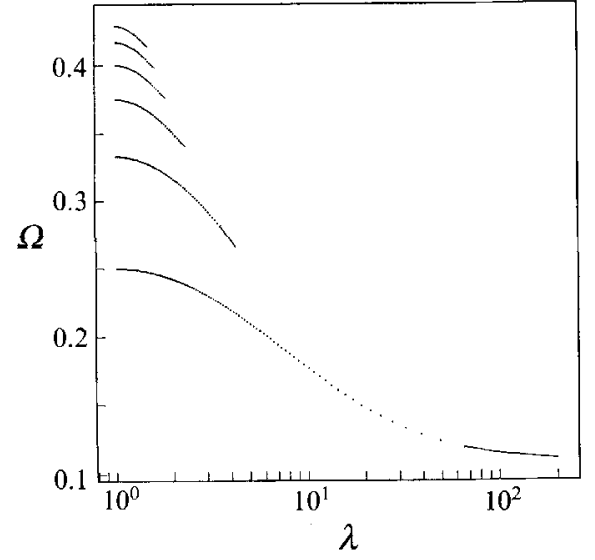

(c)

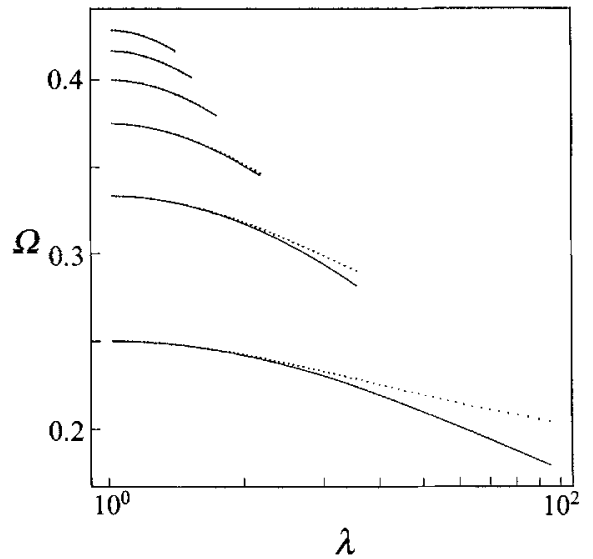


region between the largest-amplitude solid curve and the dotted curve contains the converged unstable equilibria.

Notice first that, with the exception of mode 2, the amplitude is still rather small before the waves develop cusps. This behaviour is typical of similar nonlinear vortex equilibrium problems (Polvani, Flierl \& Zabusky 1989a). Essentially, in the frame of reference rotating with the wave $m$, the flow exhibits $m$ stagnation points, located outside the vortex edge at longitudes $\varphi_{s}=2 \pi j / m(j=0, \ldots, m-1)$. It is a simple matter to show that the meridional location of the stagnation points for the linear waves are given by the simple expression:

$$
z_{s}=\frac{z_{0}-1 / m}{1-1 / m}
$$

Hence as $m$ increases, the stagnation points get increasingly close to the vortex boundary, and thus cusps develop at smaller wave amplitude. The $m=2$ wave is a notable exception, as is the case for the planar problem. The solution first bifurcates in the direction of a 'dumbbell' shape (dotted line in figure $3 a$ ), and at even larger amplitudes it further bifurcates into a two-vortex equilibrium when the neck is cut off; such equilibria are discussed in \$3.2. Although these are unforced barotropic equilibria, the presence of similar ones in the stratosphere may affect the evolution of the polar vortex during major warming events.

All the solutions in figure $3(a)$ have the same area, equal to $2 \pi\left(1-z_{0}\right)$, and thus we need only plot the angular velocity $\Omega$ versus the aspect ratio for each mode. These nonlinear dispersion relations are given in figure $3(b)$. The dotted and solid portions of each curve are used to represent the stability properties, the latter being the unstable equilibria. To each dot corresponds a stable equilibrium solution. The $m=2$ wave extends much further in amplitude (i.e. in $\lambda$ ) because it bifurcates into a peanut shape, while the higher modes are arrested by the presence of the corotating stagnation points.

As we decrease the value of $\vartheta_{0}$, nonlinear waves exist for larger amplitudes. The case $\vartheta_{0}=30^{\circ}$ is shown in figure $4(a)$. These are orthographic projections from $20^{\circ}$ latitude $0^{\circ}$ longitude. Again the $m=2$ wave extends to $\lambda \rightarrow \infty$, bifurcating ultimately into a two-vortex equilibrium. The nonlinear dispersion relations are shown in figure $4(b)$. Notice that the drop in angular velocity with amplitude is more marked at this value of $\vartheta_{0}$.

Incidentally, we have compared these numerically derived dispersion curves with the ones from weakly nonlinear theory. From Appendix A of Dritschel (1988b), it is easy to show that the weakly nonlinear dispersion relation that includes terms of order amplitude squared is given by

$$
\Omega=\frac{1}{2} \tilde{\omega}(m-1)\left[\frac{1}{m}-\frac{\left(1+z_{0}^{2}\right)}{4\left(1+z_{0}\right)^{2}} \epsilon^{2}\right],
$$

where the first term in the bracket is the linear term, cf. (9), and the wave amplitude $\epsilon$ is related to the aspect ratio via $\lambda \equiv(1-\epsilon) /(1+\epsilon)$. For the solutions with $\vartheta_{0}=30^{\circ}$, these values are plotted (solid lines) against the fully nonlinear ones (dots) in figure $4(c)$. Notice how the agreement holds to very large amplitudes.

FIgURE 4. (a) The nonlinear wave solutions for $\vartheta_{0}=30^{\circ}$. These are orthographic projections from $20^{\circ}$ latitude and $0^{\circ}$ longitude. The north pole is indicated by the cross. The dashed line is the equator. The solid lines represent stable steadily propagating solutions at progressively increasing amplitudes of the wave. The dotted lines are unstable solutions, as described in the text (see $\$ 2.5$ ). (b) Same as figure $3(b)$, but for $\vartheta_{0}=30^{\circ}$. (c) The weakly nonlinear dispersion relation (solid curves) for the $2 \leqslant m \leqslant 7$ waves at $\vartheta_{0}=30^{\circ}$, as a function of aspect ratio. The dots correspond to the numerically derived fully nonlinear values. 


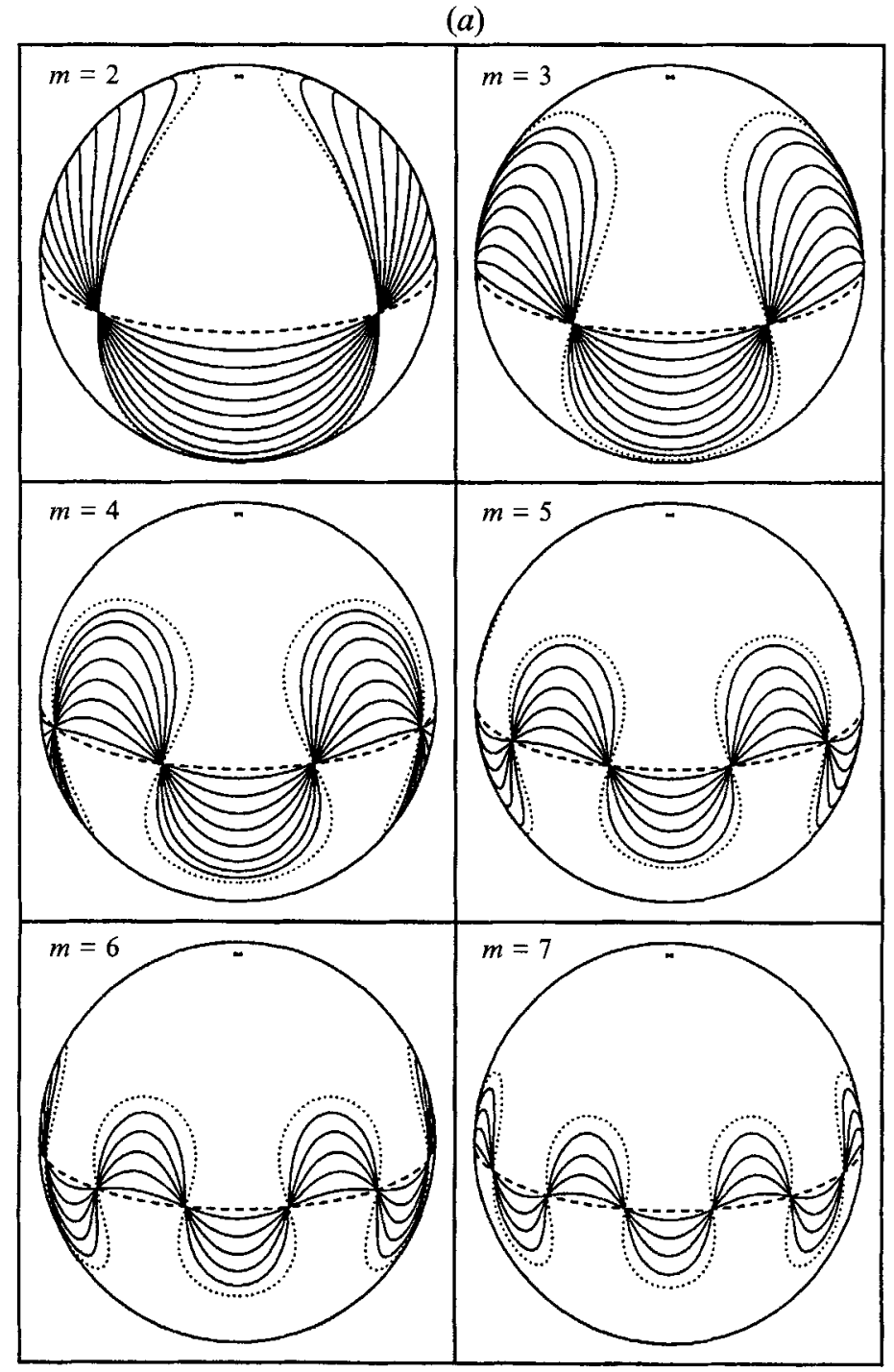

(b)

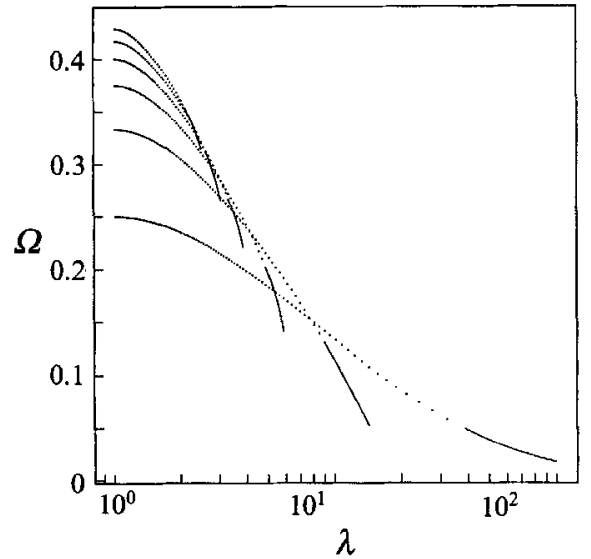

FIgURE 5. (a) Same as figure 4(a), but for $\vartheta_{0}=0^{\circ}$. (b) Same as figure $3(b)$, but for $\vartheta_{0}=0^{\circ}$. 
Lastly the equatorial solutions (i.e. $\vartheta_{0}=0^{\circ}$ ) are shown in figure 5(a). Again these are orthographic projections from $20^{\circ}$ latitude $0^{\circ}$ longitude. The corresponding nonlinear dispersion relations are given in figure $5(b)$. The fall-off of the angular velocity with amplitude is very steep for these equatorial waves.

Mode 2 is particularly interesting, since we can find solutions up to very large amplitudes and find analytically the equilibrium corresponding to $\lambda \rightarrow \infty$. This is constructed by dividing the sphere by two great circles lying in mutually orthogonal planes intersecting on the $x$-axis, and filling the different sectors with alternating values of positive and negative vorticity. The vorticity distribution is then reminiscent of a beach ball. It is also clear that such a solution exists for each mode $m$, the sphere being subdivided into $2 m$ sectors of alternating vorticity. Our algorithm, however, does not bifurcate into such beach ball equilibria for $m>2$. This is because there are a large number of other highly symmetric solutions on the sphere; these can all be constructed by filling in any pattern formed by the nodal lines of a spherical harmonic function with alternating values of vorticity (see Busse 1975). It should be clear that these equilibrium solutions are all unstable since they have cusps on their boundaries (Dritschel \& McIntyre 1990). The stability properties of the smooth equilibria are discussed next.

\subsection{Stability of the nonlinear waves}

Two main approaches have been used in the past to study the stability of equilibrium nonlinear vortex-patch solutions. The first approach (Saffman \& Schatzman 1982; Dritschel 1985) consists of doing an explicit linear stability analysis of the numerically obtained nonlinear equilibria. While this approach yields the global stability picture, it requires much labour and only yields linear results. An alternative method (Zabusky 1981; Dritschel 1985, 1989; Polvani \& Carton 1990) consists of taking initial value problems with slightly perturbed equilibria, and observing their evolution. This is computationally intensive but straightforward, and moreover yields results on the nonlinear evolution of the unstable equilibria.

We have used the second approach in this paper. The equilibrium solutions are perturbed by displacing each nodal position by a very small amount, a random number between $\delta$ and $-\delta$. This is equivalent to superimposing a large number of linear modes on the nonlinear equilibrium, and allows the most unstable mode to emerge naturally, as well as the nonlinear development to be observed. We have used the value $\delta=0.001$ for the results presented here (recall that the sphere has radius 1).

The numerical initial value problems are performed with spherical Contour Surgery (Dritschel 1988c, 1989). For stable equilibria, we typically compute dozens of periods without any noticeable change in shape; this does not of course guarantee stability. However, in the unstable cases the perturbation grows to large amplitudes in a few rotation periods. In other words, with the possible exception of the stability boundary where the growth rates could be extremely small, we have allowed enough time to see the most unstable mode emerge from the initial conditions.

The main stability results have already been mentioned. In figures $3(a), 4(a)$ and $5(a)$, the solid line are stable equilibria. The region between the dotted line and the largest-amplitude solid line is where our algorithm converged to equilibria that were found to be unstable. Notice that this region is pretty small. We suspect that the reason why our algorithm does not converge at even higher amplitude is related to the instability of the solutions it is trying to converge to. Similarly, in figures $3(b), 4(b)$ and $5(b)$ the solid portion of the lines are unstable regions, while each dot of the loweramplitude portion represents a stable converged equilibrium solution. 


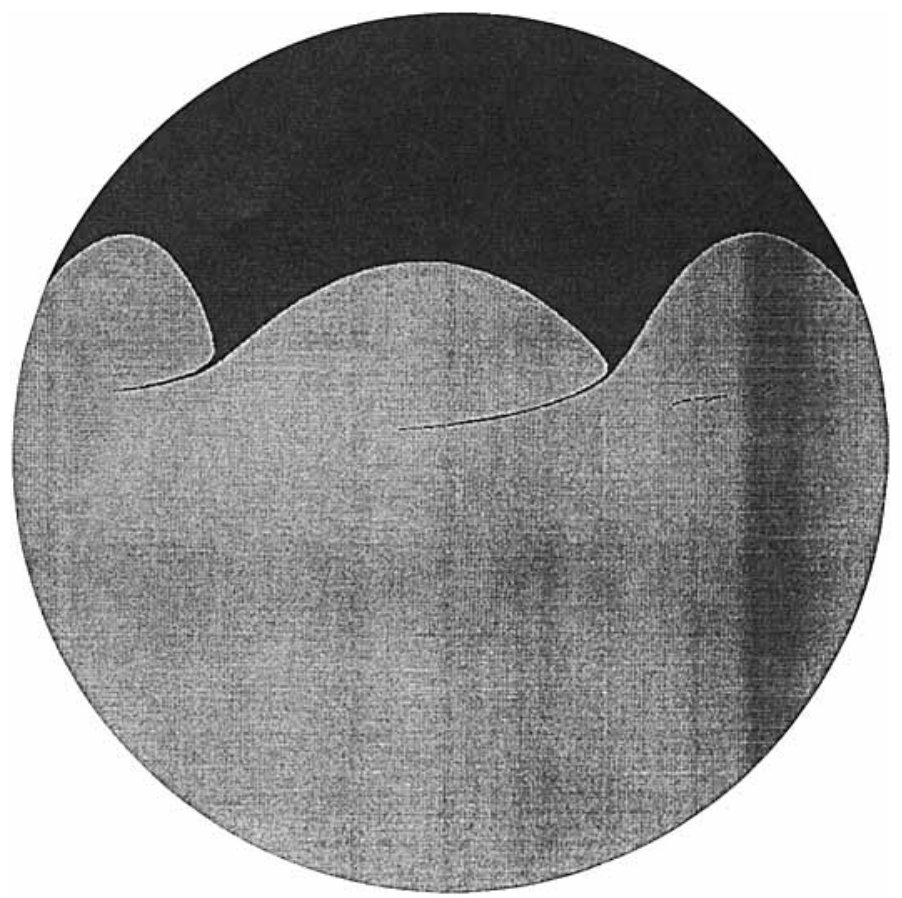

FIGURE 6. The vorticity field at $t=30$ for a randomly perturbed $m=6, \vartheta_{0}=30^{\circ}, \lambda=1.597$ wave. The non-dimensional time corresponds to approximately 12 periods of the unperturbed wave. This is an orthographic projection from $80^{\circ}$ latitude, $30^{\circ}$ longitude.

A first result that can easily be gathered from figures $3-5$ is that these nonlinear waves are stable up to considerable amplitudes. A most striking example of this is the equatorial mode- 2 wave (cf. figure $5 a$ ). The first unstable equilibrium is found near $\lambda \approx 39$; at this stage the vorticity distribution resembles a baseball; below the stability threshold, we have found such unusual shapes to be extremely stable, i.e. the nonlinear evolution consists of wavy oscillations superimposed on the baseball shape, without a major disruption of the vorticity distribution.

Secondly, we have found that instability manifests itself in two rather distinct fashions, which we have termed 'weak' and 'strong' instabilities. Weak instability is found near the cuspy solutions, and leads to the smoothing of the cusps by the ejection of thin filaments. This is typical of the high-wavenumber equilibria $(m>2)$ at $\vartheta_{0}=30^{\circ}$ and $60^{\circ}$. An example is given in figure 6 for the $m=6$ wave at $\vartheta_{0}=30^{\circ}$ with amplitude $\lambda=1.597$. (For all the figures in this section $\tilde{\omega}=2 \pi$.) We show the numerical solution at $t=30$, which corresponds to about 12 periods of the undisturbed wave (the first filaments are actually observed around $t \approx 10$ ). The reason we call this a weak instability is that the main morphology of the vorticity field remains unchanged; by shedding filaments the vortex effectively reduces slightly its aspect ratio until it becomes stable.

Strong instability, on the other hand, leads to a major rearrangement of the vorticity. This is most commonly observed for the equatorial waves at large amplitude. A typical instability is given in figure 7 , which shows the evolution of a slightly perturbed $m=5, \vartheta_{0}=0^{\circ}$ wave with $\lambda=3.444$. Notice that it takes about nine rotation periods before the instability emerges from the random perturbation field, but once the amplitude of the disturbance is sufficiently large the shedding of vortices by breakup of the lobes is quite rapid. For these equatorial waves the direction of the breaking is 

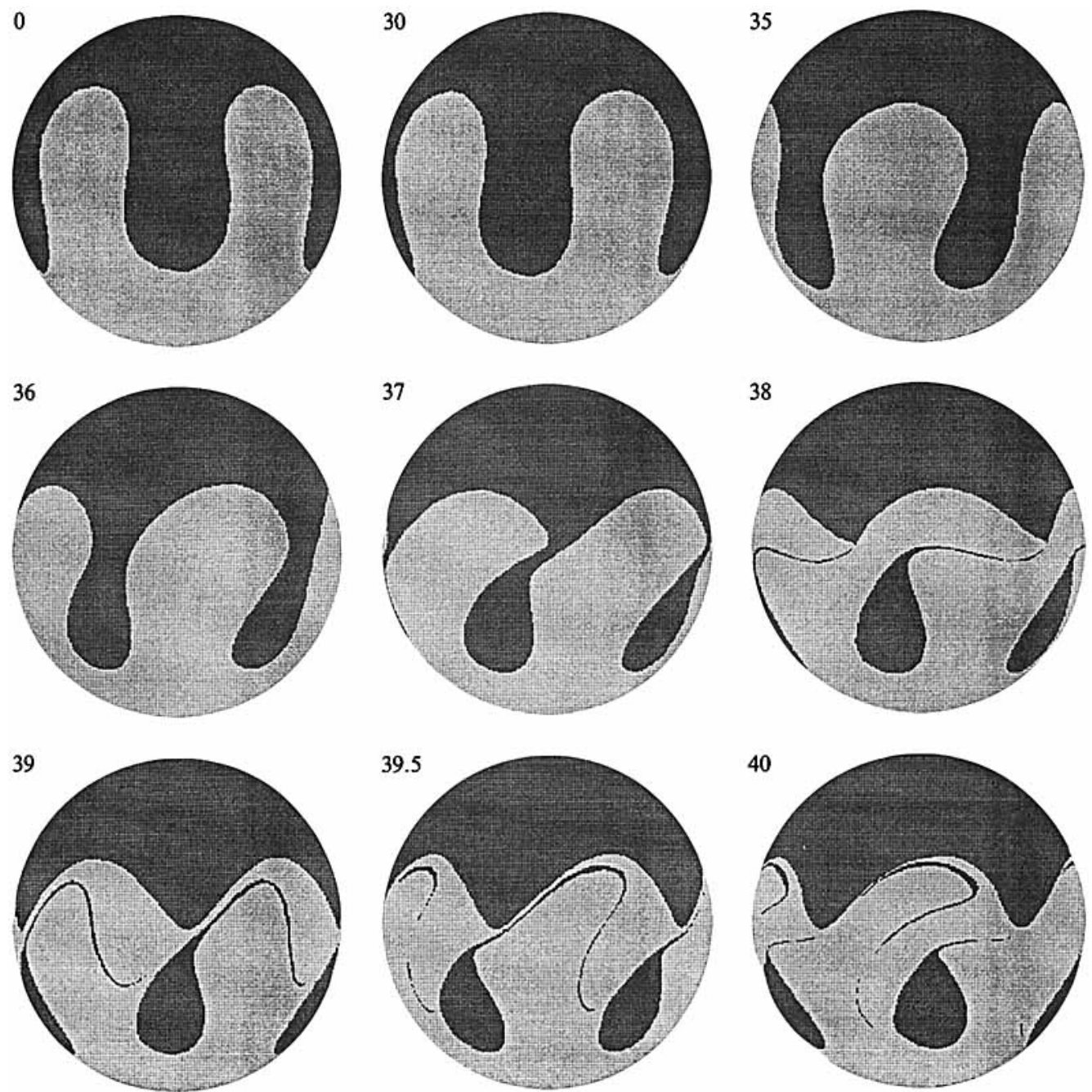

FIGURE 7. The evolution of an initially perturbed $m=5, \vartheta_{0}=0^{\circ}$ wave with $\lambda=3.444$. The numbers beside each frame indicates the time. The rotation period of the unperturbed nonlinear wave is approximately 3.860 .

very much dependent on the initial conditions, as is evidenced by the case shown in figure 8 . Here a randomly perturbed $m=7, \vartheta_{0}=0^{\circ}$ wave with $\lambda=2.333$ breaks inward, leading to the entrainment of low-vorticity fluid into the northern polar cap. For the $\vartheta_{0}=30^{\circ}$ and $60^{\circ}$ waves however, the breaking always occurs outwards, i.e. the lobes of the waves pinch-off patches of high vorticity in the south of the polar cap. This is because the shear is always greater equatorward (Dritschel 1988 b, 1989). The fact that patches, rather than filaments, are pinched-off is related to the general instability of strips of vorticity located in equatorial regions - basically, the shear is not adequate to elongate the lobes and roll-up occurs instead (see Dritschel \& Polvani 1992 for further remarks).

We have found that for mildly supercritical amplitudes the most unstable modes are symmetric ones, as exemplified by figures 7 and 8 . At larger supercritical amplitudes, however, the evolution is asymmetric. An example of this is given in figure 9 , for the $m=5, \vartheta_{0}=0^{\circ}$ wave at $\lambda=3.878$. Again, notice that it takes more than three wave 

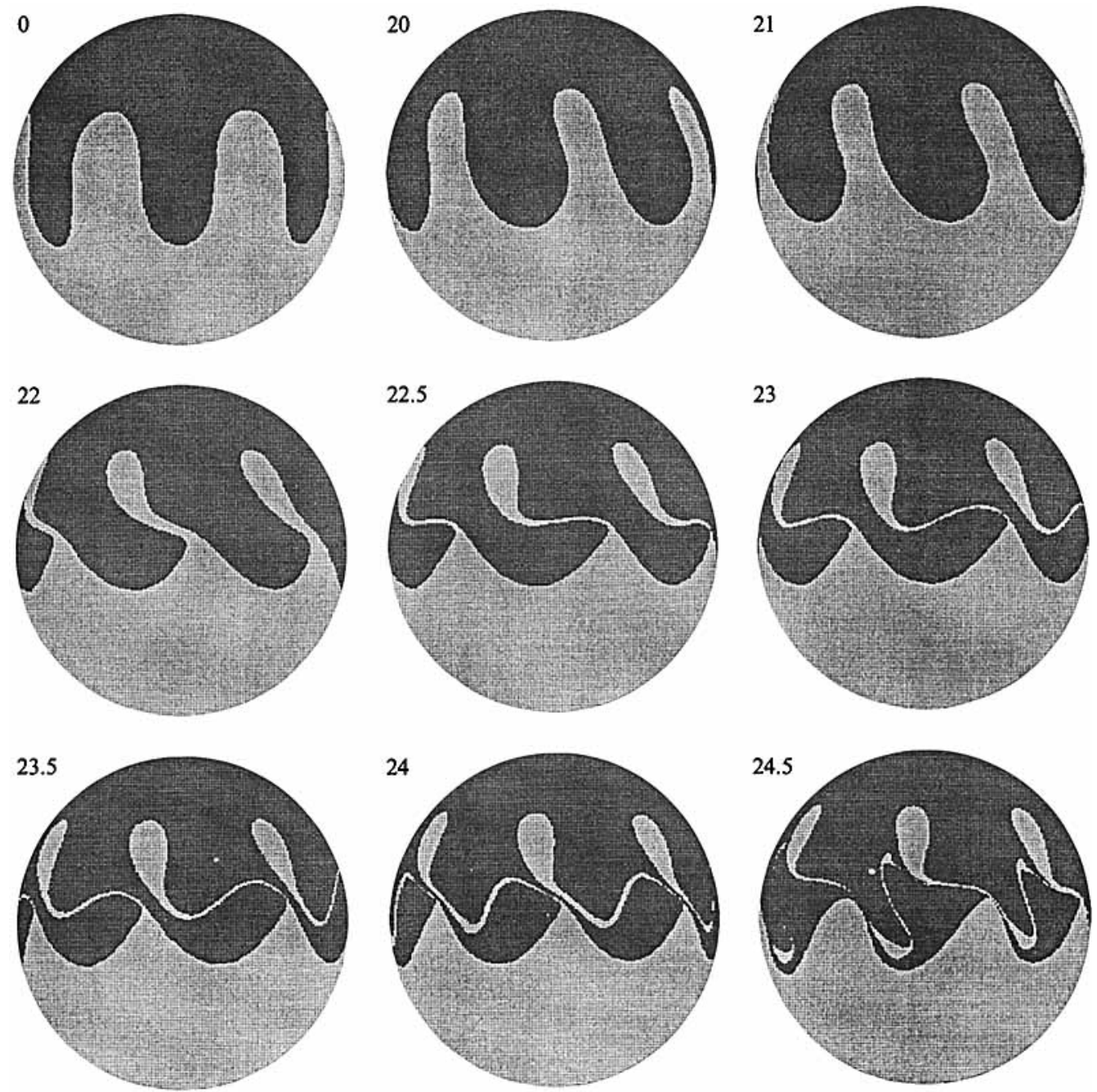

FIGURE 8. Same as figure 7 but for an $m=7, \vartheta_{0}=0^{\circ}$ wave with $\lambda=2.333$. The rotation period of the unperturbed nonlinear wave is approximately 2.995 .

rotation periods before the instability emerges, but after that the catastrophic disruption of the vorticity field is comparatively fast. Notice how the lobes of the finiteamplitude unstable waves pinch-off and merge (cf. frames $t=16.5,17$, and 17.5 of figure 9).

The $m=2$ waves exhibits the most peculiar behaviour. As we have already pointed out (cf. figure $5 a$ ) the equatorial waves $m=2$ are stable to extremely large amplitudes, in which the vorticity distribution resembles a baseball. The stability boundary is found at $\lambda \approx 39$. The nonlinear evolution of the unstable equilibria is, however, remarkable. It should be classified as 'strong' following the definition adopted above, were it not for the fact that the initial state periodically recurs. An example for the equilibrium at $\lambda=65.667$ is shown in figure 10 . The instability, which disrupts the original shape at about one-third of the unstable equilibrium rotation period $(t \approx 12)$, leads to an oscillation of the vorticity distribution between two very simple unstable states: a 'beach-ball' state (cf. $t=21,47.5,91.5$ of figure 10), and a state where the positive vorticity is confined to two elliptical polar caps (cf. $t=14.5,67.5$ in figure 10). 

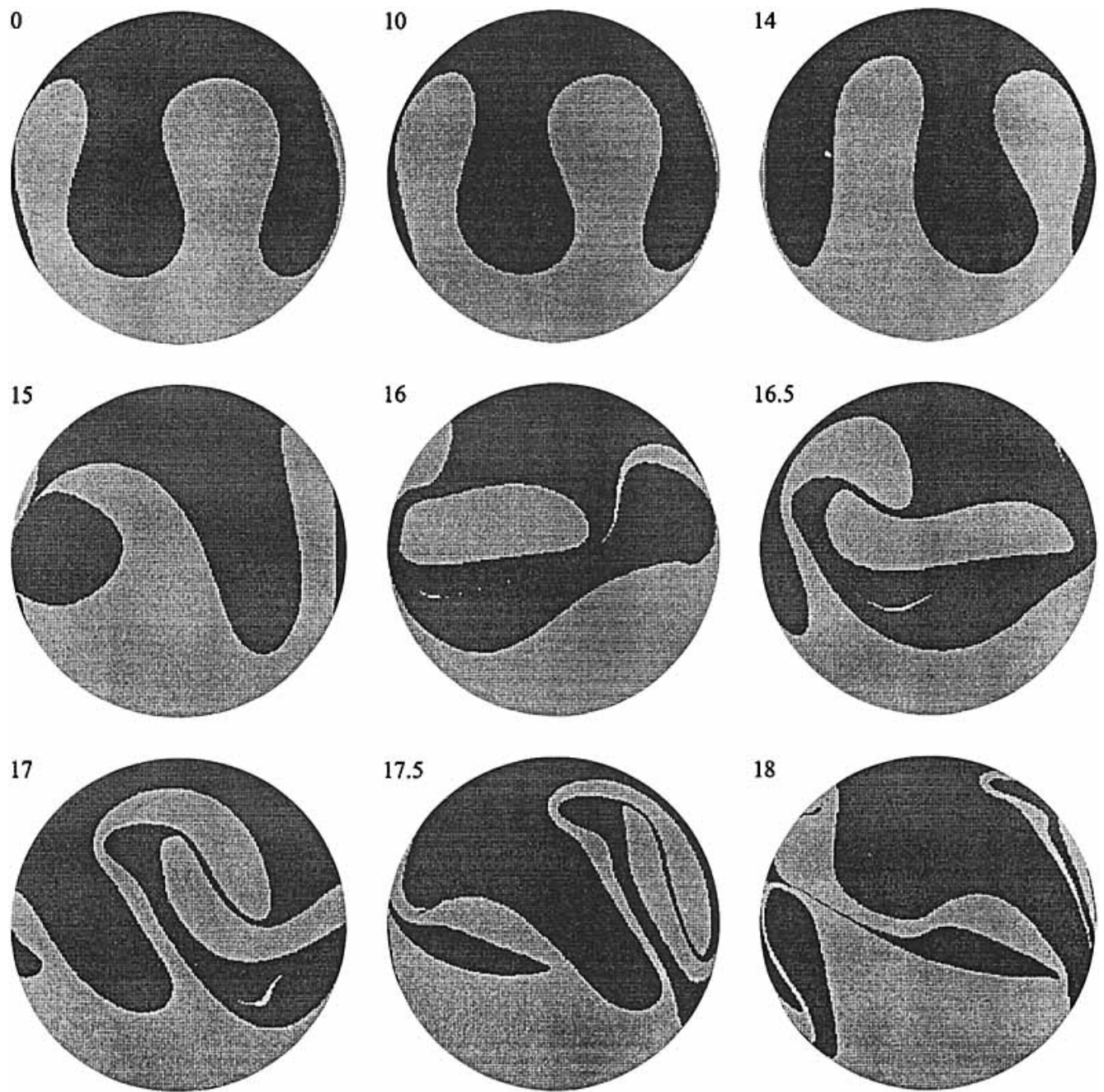

FIGURE 9. Same as figure 7 but for an $m=5, \vartheta_{0}=0^{\circ}$ wave with $\lambda=3.878$. The rotation period of the unperturbed nonlinear wave is approximately 4.520 .

Finally, the instability of the $\vartheta_{0}=60, m=2$ equilibria reveals a behaviour that may be important for the dynamics of the stratospheric polar vortex during major warmings. This is illustrated in figure 11, depicting the evolution of a slightly perturbed equilibrium $(\lambda=65.667)$. After about one rotation period, the instability splits the vortex into two pieces that rotate around the pole without merging. The relative constancy of the distance between the two vortices as well as their shapes strongly suggests that there exist equilibria composed of more than one vortex. They are the subject of the next section.

\section{Multi-vortex equilibria on the sphere}

\subsection{Point-vortex equilibria and their stability}

Before considering the equilibrium shapes and stability properties of arrays of corotating finite-area vortices on the spherical surface, we derive the analogous results for arrays of point vortices. The corresponding problem for the planar geometry is 

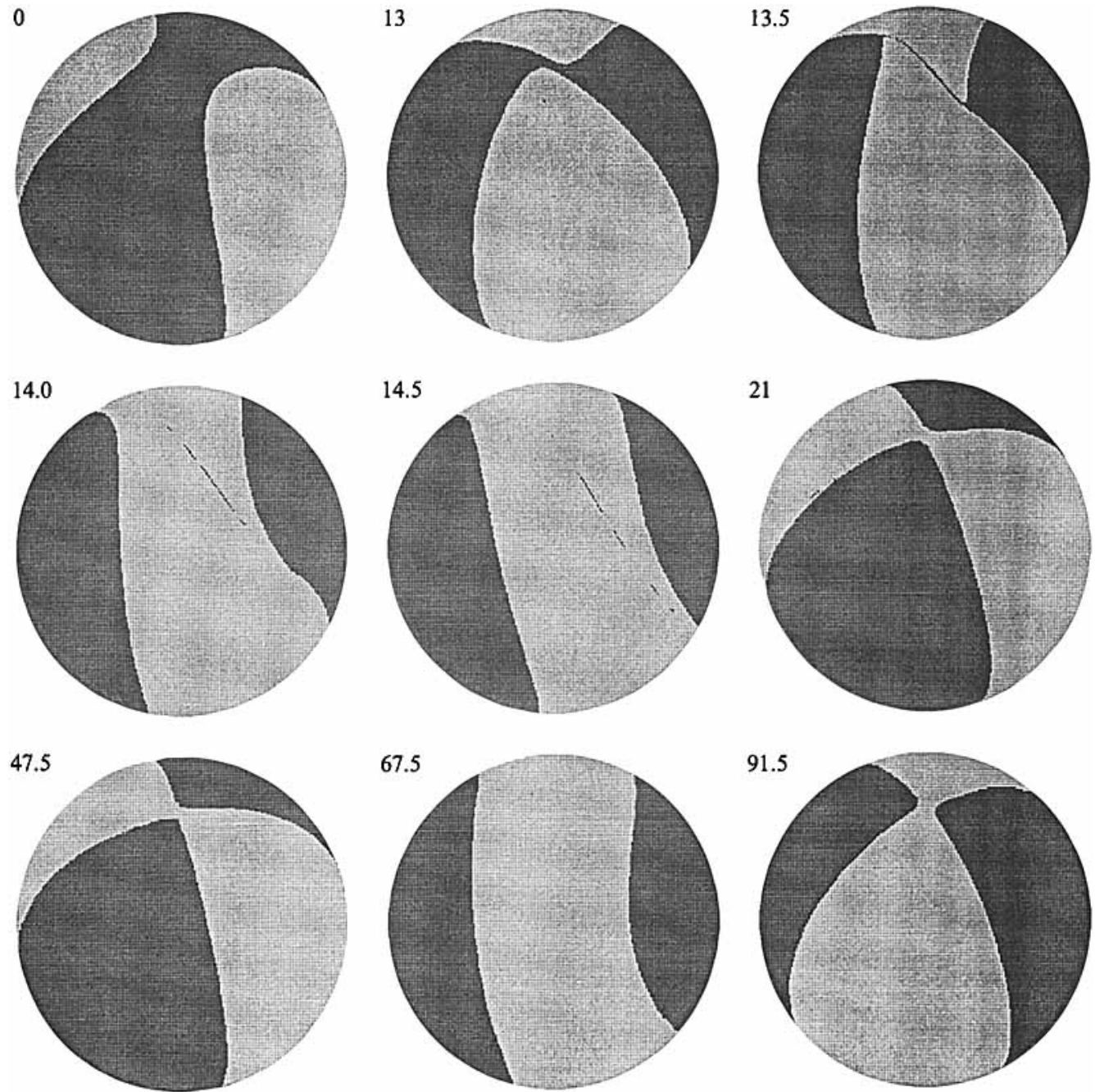

FIgURE 10. Same as figure 7 but for an $m=2$, equatorial wave with $\lambda=65.667$. The rotation period of the unperturbed nonlinear wave is approximately 34.885 .

a century old; Thomson (1883) discovered that $N$ corotating point vortices of equal strength are (linearly) stable for $N \leqslant 7$. The finite-area solutions for $N=2$ were found by Saffman \& Szeto (1980) and for $2 \leqslant N<8$ by Dritschel (1985). The main stability result for the finite-area vortices is that instability occurs at any $N$ when the area of the vortices becomes sufficiently large; loosely speaking, when the vortices are nearly touching the configuration is almost always unstable (when $N=7$, any finite size leads to instability - see Dhanak 1992). We now extend these results to spherical geometry.

Consider first a single point vortex of strength $\kappa$, located at $\boldsymbol{x}^{\prime}$ on a spherical surface. The stream function $\psi$ it induces at any point $\boldsymbol{x}$ on the sphere is given by the simple expression

$$
\psi(x)=\frac{1}{2} \kappa \log \left(1-x \cdot x^{\prime}\right) .
$$

Recall that the strength $\kappa$ is defined by $\kappa=\Gamma / 2 \pi$ where $\Gamma$ is the circulation. It is important to notice that the form (15) includes a constant vorticity of value $-\frac{1}{2} \kappa$ spread evenly over the spherical surface; this is necessary to ensure that the global circulation 

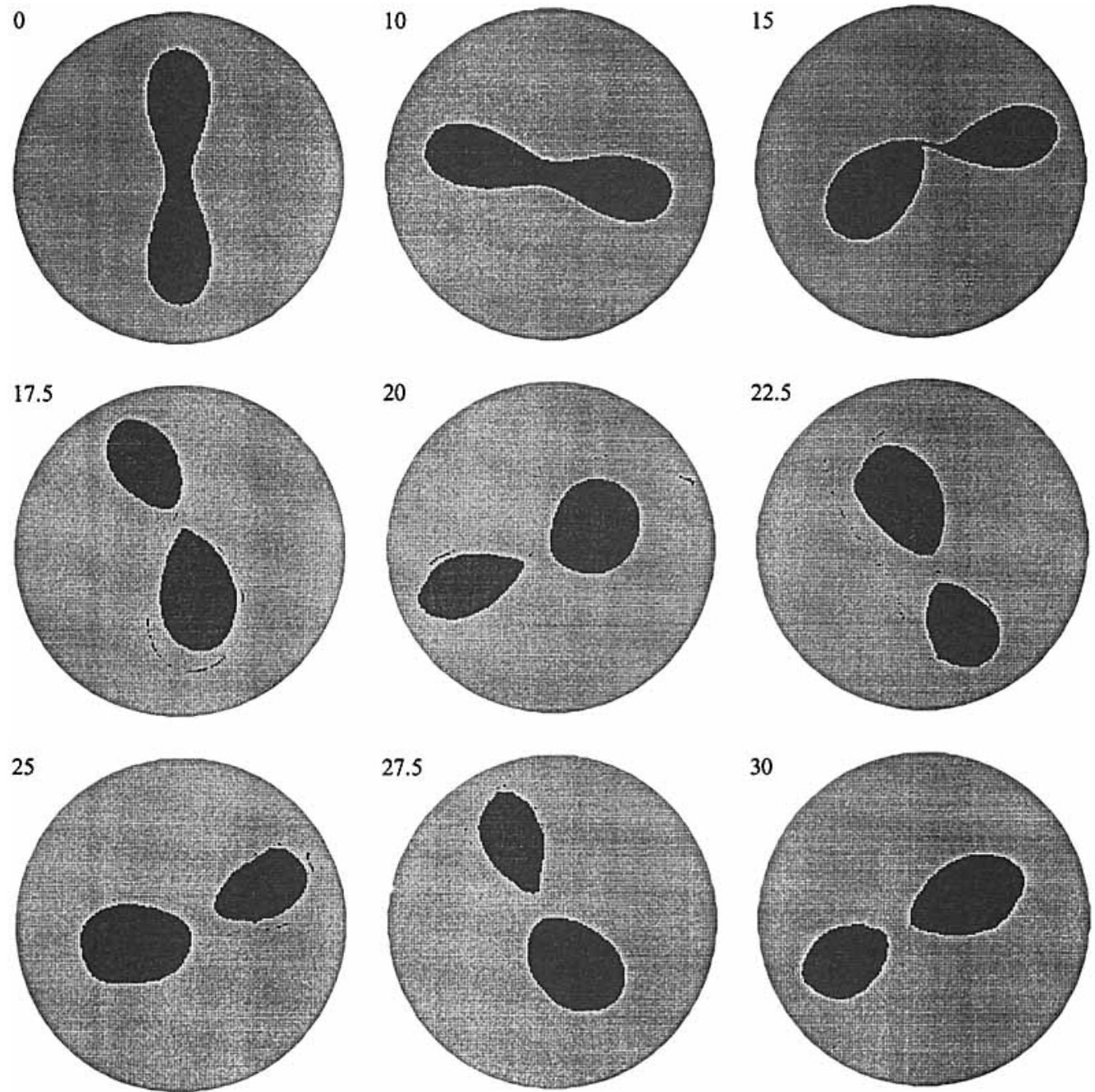

FIGURE 11. Same as figure 7 but for an $m=2, \vartheta_{0}=60^{\circ}$ wave with $\lambda=65.667$. The rotation period of the unperturbed nonlinear wave is approximately 8.222 .

is equal to zero. The analogous expression in latitude and longitude coordinates is found in Kimura \& Okamoto (1987).

Consider next $N$ point vortices located at positions $x_{k}(k=0, \ldots, N-1)$ on the sphere, and with strengths $\kappa_{k}$. The equations of motion for this system take a very simple form in Cartesian coordinates. Substituting (15) into (4) yields immediately:

$$
\frac{\mathrm{d} \boldsymbol{x}_{k}}{\mathrm{~d} t}=\frac{1}{2} \sum_{j \neq k} \kappa_{j} \frac{\boldsymbol{x}_{j} \times \boldsymbol{x}_{k}}{1-\boldsymbol{x}_{j} \cdot \boldsymbol{x}_{k}} .
$$

We now limit our attention to the steadily rotating configuration constituted by $N$ equidistant point vortices located on the same latitudinal circle $z=z_{0}$, and with identical strength $\kappa$. This equilibrium is an exact solution of (16), with

$$
x_{k}=\left(r_{0} \cos \varphi_{0 k}, r_{0} \sin \varphi_{0 k}, z_{0}\right), \quad \varphi_{0 k}=2 \pi k / N, \quad k=0, \ldots, N-1,
$$

where $r_{0}=\left(1-z_{0}^{2}\right)^{\frac{1}{2}}$. The angular velocity $\Omega$ with which this array of point vortices rotates is given by

$$
\Omega=(N-1) \frac{1}{2} \kappa z_{0} / r_{0}^{2}=z_{0} \Omega_{p},
$$




\begin{tabular}{rll}
$N$ & $\mathrm{z}_{\mathrm{c}}$ & \multicolumn{1}{c}{$\begin{array}{c}\vartheta_{c} \text { (deg.) } \\
\text { always stable }\end{array}$} \\
2 & & $\begin{array}{l}\text { always stable } \\
3\end{array}$ \\
4 & $1 / \sqrt{ } 3$ & $35.26438968 \ldots$ \\
5 & $1 / \sqrt{ } 2$ & 45 \\
6 & $2 / \sqrt{ } 5$ & $63.43494882 \ldots$ \\
7 & 1 & 90 \\
$>7$ & & always unstable
\end{tabular}

TABLE 1. The critical values of $z$ and latitude, $z_{c}$ and $\vartheta_{c}$ respectively, equatorward of which an array of $N$ corotating colatitudinal point vortices of equal strength become unstable on a spherical surface

where $\Omega_{p}$ is the angular velocity of $N$ corotating point vortices on the plane. Hence $\Omega$ decreases monotonically with latitude. Near the pole $\left(z_{0} \rightarrow 1\right)$ the planar value is recovered. More interestingly, $\Omega$ vanishes when the point vortices are located on the equator (or indeed any great circle around the sphere).

The stability analysis for this configuration is messy but not intrinsically difficult. We limit ourselves to sketching the main points of the derivation. As pointed out in $\S 2.1$, the variable conjugate to the Cartesian vertical coordinate $z$ is the longitude $\varphi$. From (16), one can write the evolution equation for these two variables:

$$
\frac{\mathrm{d} z_{k}}{\mathrm{~d} t}=\frac{1}{2} \kappa \sum_{j \neq k} \frac{r_{j} r_{k} \sin \left(\varphi_{k}-\varphi_{j}\right)}{1-\boldsymbol{x}_{j} \cdot \boldsymbol{x}_{k}} \text { and } r_{k} \frac{\mathrm{d} \varphi_{k}}{\mathrm{~d} t}=\frac{1}{2} \kappa \sum_{j \neq k} \frac{z_{j} r_{k}-z_{k} r_{j} \cos \left(\varphi_{k}-\varphi_{j}\right)}{1-\boldsymbol{x}_{j} \cdot \boldsymbol{x}_{k}}
$$

where $r_{i}=\left(1-z_{i}^{2}\right)^{\frac{1}{2}}$, and $1-x_{j} \cdot x_{k}=1-z_{j} z_{k}-r_{j} r_{k} \cos \left(\varphi_{k}-\varphi_{j}\right)$. By analogy with the planar problem, the perturbed vortex position for the $k$ th vortex is written in the form (see Dritschel 1985)

$$
z_{k}=z_{0}+\zeta r_{0}^{2} \omega_{p k} \mathrm{e}^{\sigma t} \text { and } \varphi_{k}=\varphi_{0 k}+\xi \omega_{p k} \mathrm{e}^{\sigma t}
$$

where $\omega_{p k} \equiv \mathrm{e}^{2 \pi \mathrm{i} p k / N}$ and $p$ indicates the symmetry of the mode, $p=0,1, \ldots,\left[\frac{1}{2} N\right]$ (here [] indicates 'integer value of'), and $\zeta$ and $\xi$ are infinitesimal constant amplitudes. Substitution of these expression into (18) and linearization yields a standard eigenvalue problem for the growth rate $\sigma$. After much algebra, one obtains the following dispersion relation:

$$
\left(\sigma r_{0}^{2}\right)^{2}=(p-1)^{2}(p+1-N)^{2}-(N-1)^{2}-r_{0}^{2}(N-1)[(p-1)(p+1-N)-(N-1)] .
$$

The general result from this formula is that point vortex arrays are more unstable on the sphere than in the plane. At the pole, $N<7$ is stable, $N=7$ is neutral and $N>7$ is unstable, in accordance with the planar results. Away from the pole, $N=7$ is always unstable and, as the equilibrium latitude of the vortex array is decreased, $N=6$ first become unstable equatorward of approximately $63^{\circ}, N=5$ equatorward of $45^{\circ}$, $N=4$ equatorward of approximately $35^{\circ}$, so that, on the equator itself only $N=2$ and 3 are stable. The critical values of $z$ are summarized in table 1 . We have numerically verified them by direct numerical integration of (16). With this in mind we now proceed to considering the finite-area problem.

\subsection{Finite-area multi-vortex equilibria and their stability}

Knowing the point-vortex results, we now determine the finite-area multi-vortex equilibria, and study their stability. The algorithm used to determine the equilibrium shapes is quite similar to the one described in $\$ 2.3$, and the relevant details are given 


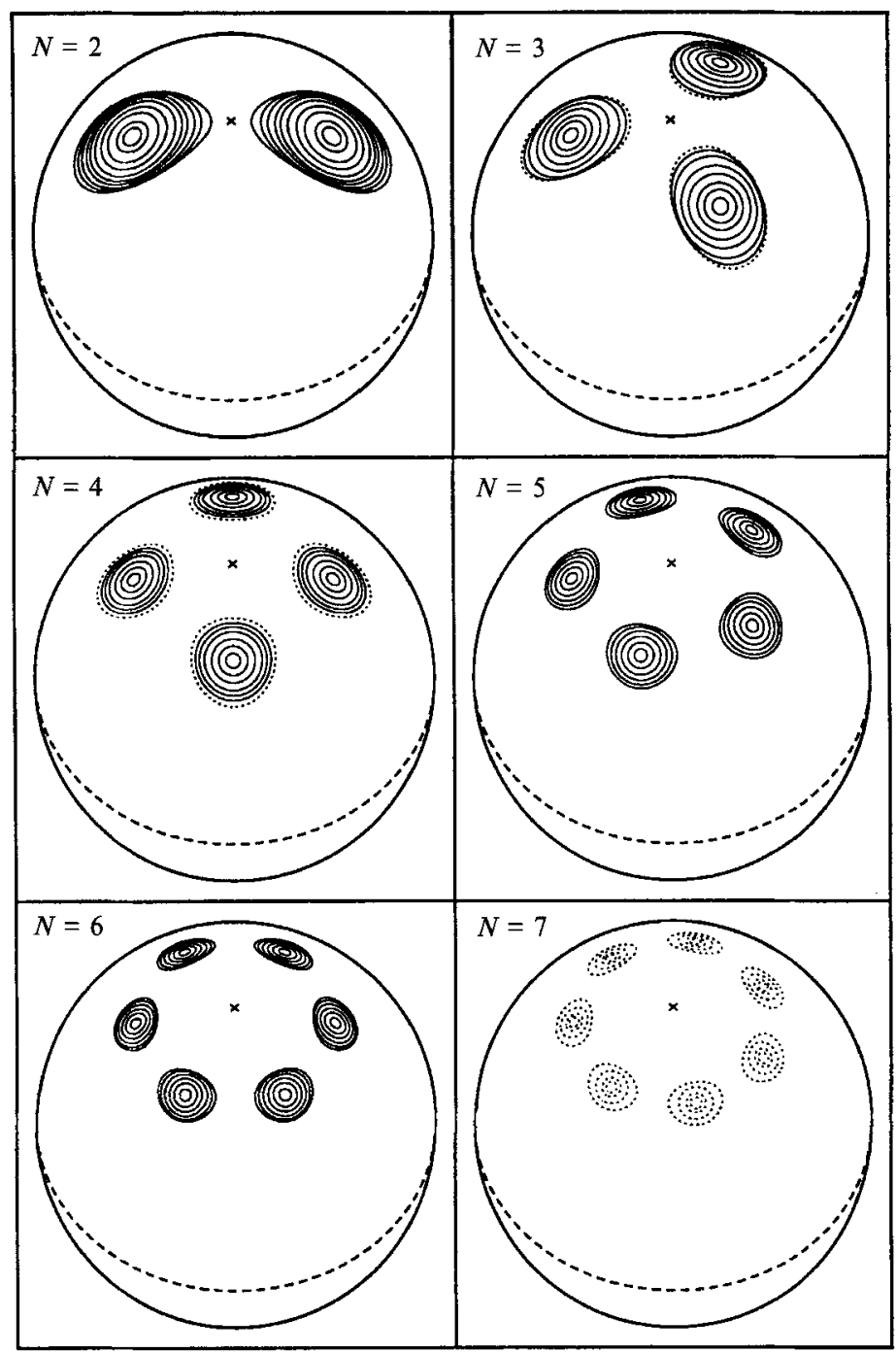

FIGURE 12. Finite-area multiple-vortex equilibria for $\vartheta_{0}=60^{\circ}$. This is an orthographic projection from $65^{\circ}$ latitude and $90^{\circ}$ longitude. The cross indicates the pole, and the dashed line the equator. Solid (dotted) lines indicate stable (unstable) equilibria (cf. §3.2).

in Appendix B. Each multi-vortex equilibrium composed of $N$ vortices is uniquely defined by two parameters: the latitude $\vartheta_{0}$ of vorticity centroid and the fractional area $\mu$, defined as the total area enclosed by the vortex patches divided by $4 \pi$. The algorithm proceeds at fixed $\vartheta_{0}$ by progressively increasing $\mu$ until convergence can no longer be achieved within several hundred iterations.

Consider first the equilibria for $\vartheta_{0}=60^{\circ}$ and $2 \leqslant N \leqslant 7$, shown in orthographic projection in figure 12. To make the presentation more concise, we use different line patterns to indicate stability; solid (dashed) lines indicate stable (unstable) solutions; moreover, although for clarity only a selected number of solutions are shown, the largest-amplitude solid (dashed) line corresponds to the largest-amplitude stable (unstable) equilibrium to which we have been able to converge. The stability was tested via initial value problems, as described in $\S 2.5$. It should be repeated that by stability, 


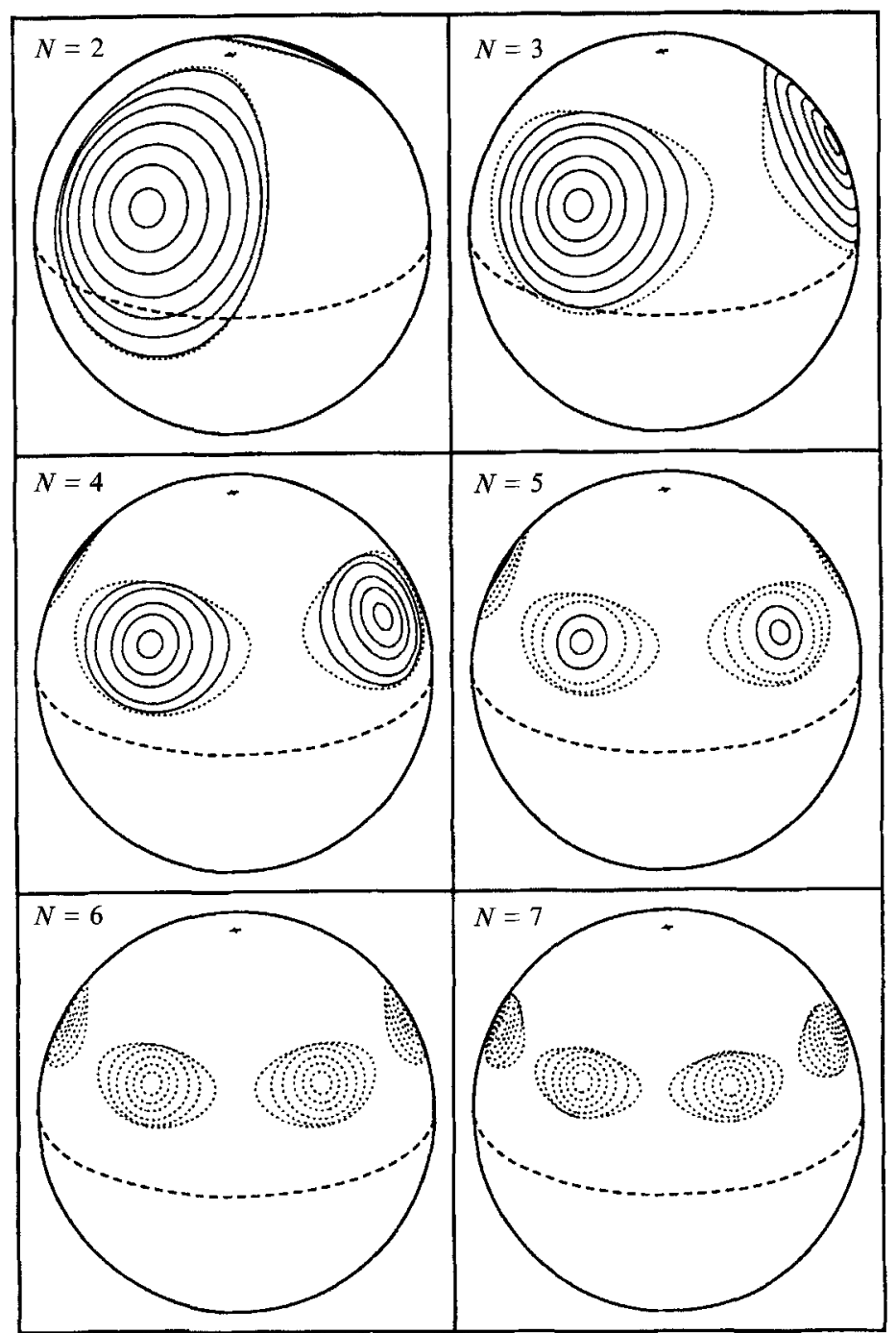

FIGURE 13. Same as figure 12, but for $\vartheta_{0}=30^{\circ}$. The orthographic projection here is from $35^{\circ}$ latitude and $30^{\circ}$ longitude.

we really mean robustness, i.e. whether the perturbed vortex arrays maintain their coherence, or whether the vortices stray far from their relative initial positions and merge.

With respect to figure 12 , it is interesting to note that, while six point vortices are linearly unstable at $\vartheta_{0}=60^{\circ}$ (cf. table 1), the corresponding finite-area equilibria are robust, at least up to a certain amplitude. The evolution of slightly perturbed $N=6$ solutions shows that the vortices alternately arrange themselves along a circle and along the sides of a 'triangle', but the basic morphology of the equilibrium is not changed. This is in contrast with the unstable solutions (say for $N=7$ ), where slightly perturbed vortices wander very far from their respective positions and repeatedly merge.

Although we have tried a number of variations on our algorithm, for most values of $N$ it has not converged for large $\mu$, i.e. when the vortices are nearly touching (cf. figure 


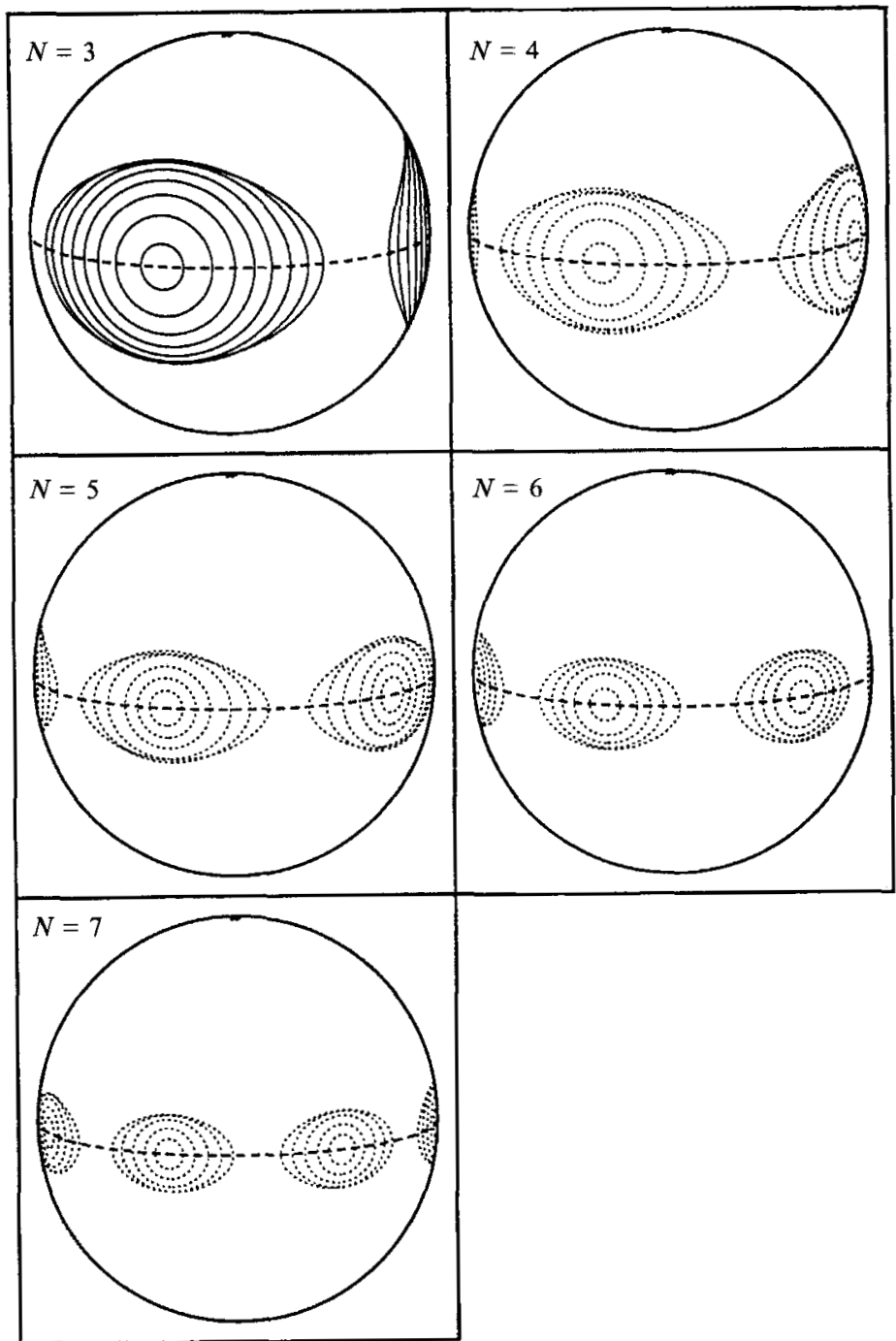

FiguRE 14. Same as figure 12 , but for $\vartheta_{0}=30^{\circ}$. The orthographic projection here is from $10^{\circ}$ latitude and $20^{\circ}$ longitude.

12). This must be because these solutions are almost certainly unstable (as is well known to happen in the planar case, Dritschel 1985). For $N=3$ and 4, the algorithm stopped converging immediately after the stability boundary was reached. It is safe to speculate that for $N=2,5$ and 6 , the stability boundary is probably not very far from the largest stable solution we have found.

Decreasing the latitude makes things more unstable, as one would expect from the point-vortex results. Figure 13 shows the $\vartheta_{0}=30^{\circ}$ solutions. At this latitude all the stability boundaries have been determined. Again, it is notable that, for small areas at least, four and five finite-area vortex arrays are robust, although four and five point vortices are linearly unstable.

The equatorial multi-vortex equilibria are presented in figure 14. In agreement with the point-vortex result, only the $N=2$ and 3 equatorial equilibria are stable. The twovortex solution is not shown, since it is known analytically; two polar caps bounded 

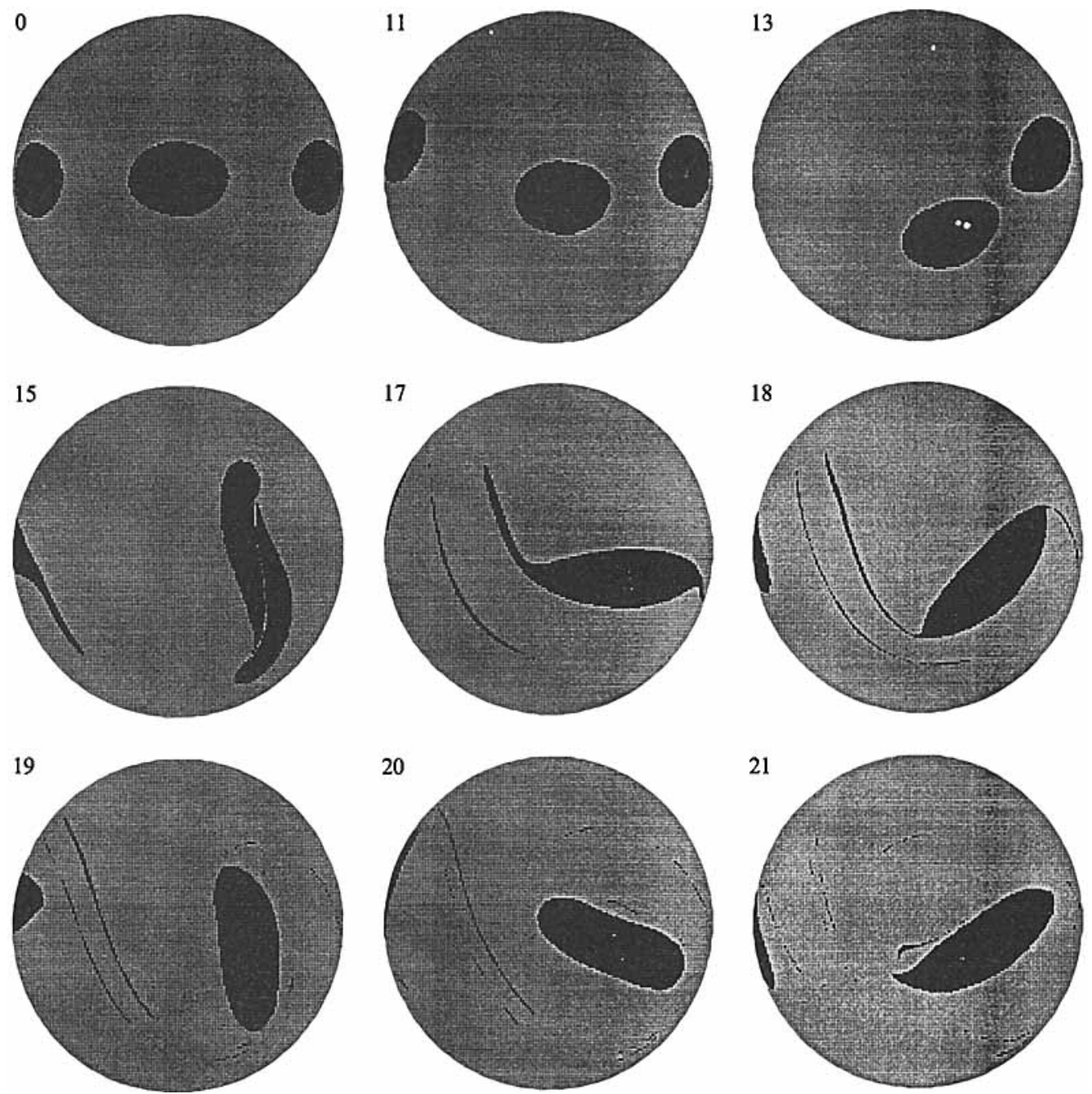

FIGURE 15 . The evolution of the slightly perturbed $N=6$ equilibrium with $\mu=0.1$.

The six vortices merge pairwise.

by circular contours is an exact solution. Even this becomes unstable for sufficiently large $\mu$. The linear stability of the $N=2$ configuration can be worked out from Dritschel \& Polvani (1992); it is easy to show that instability sets in for

$$
\mu>\sqrt{ } 2-1=0.4142136 \ldots
$$

Unfortunately, the algorithm was unable to reach the expected stability boundary for $N=3$ at large $\mu$.

These equatorial arrays are very reminiscent of vortex streets, especially for larger values of $N$. Indeed their instability also proceeds in a similar fashion, i.e. by vortex pairing and merger. A typical example for the equatorial $N=6, \mu=0.1$ equilibrium is shown in figure 15. Notice that the vortex produced after the merger is far from circular, and moreover shows no tendency for axisymmetrization.

For completeness, the variation in angular velocity $\Omega$ as a function of the fractional area $\mu$ is shown in figure 16 . Since $\Omega \equiv 0$ for all equatorial equilibria, only the cases 
(a)

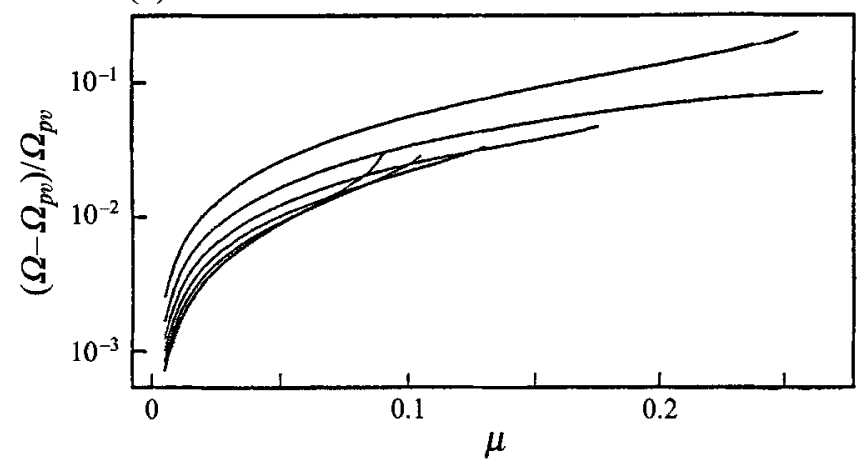

(b)

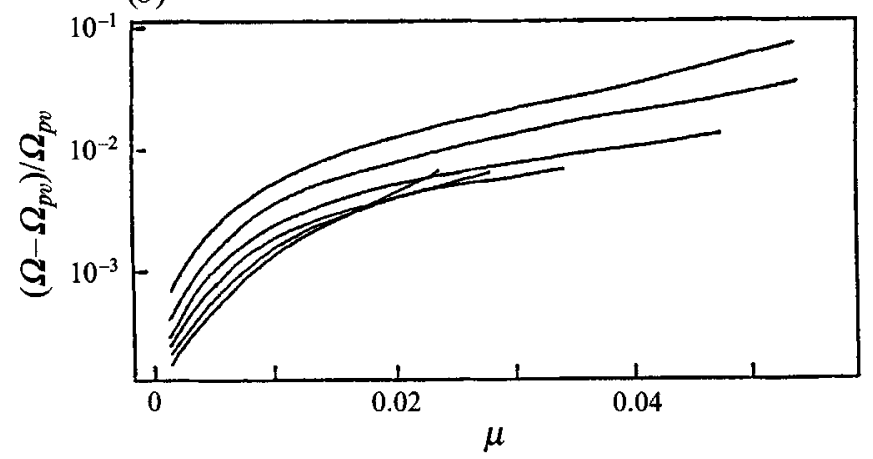

FIGURE 16. The fractional variation in angular velocity $\Omega$ compared to the equivalent point-vortex value $\Omega_{p v}$ (given by (17)) versus the $\mu$, the percentage of the area of the sphere covered by the equilibrium vortices. (a) $\vartheta=30^{\circ},(b) \vartheta=60^{\circ}$.

$\vartheta_{0}=30^{\circ}$ and $60^{\circ}$ are shown. Moreover, for each finite-area equilibrium, we can define the quantity $\Omega_{p v}$ as the angular velocity of a point-vortex array whose vortices have same circulation (computed with (17)). Since the difference between $\Omega$ and $\Omega_{p v}$ is usually small, we plot the fractional difference $\left(\Omega-\Omega_{p v}\right) / \Omega_{p v}$. As can be seen from figure 16, the main conclusion is that finite-area vortex equilibria rotate faster than their point-vortex counterparts, although the fractional difference is never more than about $10 \%$, at least for the solutions we have obtained.

\section{Summary and applications}

In this paper, we have examined two of the simplest non-axisymmetric equilibrium flows on the surface of a sphere, flows consisting of just two uniform values of vorticity separated by either a single interface or by several identical disjoint contours. We have determined their equilibrium configurations, and have examined their (nonlinear) stability by direct, high-precision, long-time numerical integration. We find that extensive sets of the equilibrium solutions are stable. In the case of a single interface, the steadily propagating waves are generally stable to large amplitude, i.e. so that the flow is very far from zonally symmetric. The most important form of instability manifests itself when the very large-amplitude waves pinch-off to produce isolated vortices.

In the case of several vortices, we have shown that colatitudinal point-vortex arrays are more unstable on a sphere than on a plane. In particular, while six or fewer vortices 
are stable at high latitudes, only three vortex equilibria are stable on the equator; this result is also valid for finite-area equilibria. This study thus confirms one of the findings of our previous study on the roll-up of vortex strips on the sphere (Dritschel \& Polvani 1992), namely that barotropic vorticity configurations tend to be more unstable near the equator than near the poles.

In venturing an application to the Earth's stratosphere, one has to admit that the neglected effects of radiation and vertical structure distance somewhat this idealized model from the real thing. However, certain features of the complex dynamics of the polar vortex can be captured by the very simple model shown in figure 1. In particular, it is worth examining what values one obtains from the dispersion relation (9) for conditions similar to the Earth's stratosphere.

Following Juckes \& McIntyre (1987), we take $\omega_{N}=2.6 \Omega_{e}$, and $\vartheta_{0}=55^{\circ}$. These choices give, from (5), a reasonable peak westerly wind speed (as measured on the Earth) of $115 \mathrm{~m} / \mathrm{s}$ at the edge of the vortex. With these parameters describing the undisturbed flow, the angular velocities as well as the periods for these waves are given in table 2 (the values are computed in the standard meterological frame of reference, i.e. as they would be measured by a observer rotating with the Earth's velocity $\Omega_{e}$ ).

These numbers are only indicative, since a barotropic uniform-vorticity cap is a very crude model of the polar vortex. However, it is interesting to note that, for sufficiently large wavenumber (in this case $m>3$ ), the westerly mean flow dominates the Rossby wave propagation mechanism, leading to eastward motion, while for the gravest modes one finds westward propagation typical of Rossby waves. The $m=3$ wave is closest to being stationary in the Earth's frame of reference.

This quasi-stationary character of the $m=3$ wave (and $m=4$ to some extent) would suggest that, under forcing conditions similar to the ones present in the stratosphere, it would be the most 'resonant' mode, i.e. the one that would extract the most energy from stationary waves perturbing the vortex from below. This has in fact been repeatedly observed in high-resolution numerical models of the stratospheric circulation. For a very clean example the reader is referred to figure F9 of the review by McIntyre (1991), where the polar vortex appears with an unmistakeably triangular shape. That figure comes from a high-resolution shallow-water model, but similar results have been found in barotropic simulations (Juckes \& McIntyre 1987; Waugh 1992). The analysis of the total ozone in the stratosphere using the TOMS spectrometer also show that, before breaking, the polar vortex often assumes a triangular form (see, for instance, figure 1 of Bowman 1992).

In studying the nonlinear regime, we have shown that once a polar vortex is sufficiently elongated it becomes dynamically unstable (cf. figure 11). This suggests that planetary wave forcing may be crucial only in the initial phases of a major warming event, in providing the elongation of the vortex, with the subsequent evolution being the consequence of simple barotropic instability. Moreover, the existence of stable twovortex equilibria probably plays the role of an attractor in affecting its evolution.

The simple barotropic model in spherical geometry may well have interesting applications to the atmospheres of the outer planets. A particularly relevant one is the so-called 'ribbon' on Saturn. This feature, centred around $46^{\circ} \mathrm{N}$, resembles a wavy black line embedded in a white background (see Ingersoll et al. 1984 for a review). There is evidence (Smith et al. 1982) that the circulation is cyclonic north of the ribbon and anticyclonic south of it, suggesting that the ribbon itself is a region of very strong vorticity gradients.

Sromovsky et al. (1983) have carefully analysed the shape of the ribbon, and extracted from the data the phase velocity of waves propagating on the ribbon as a 


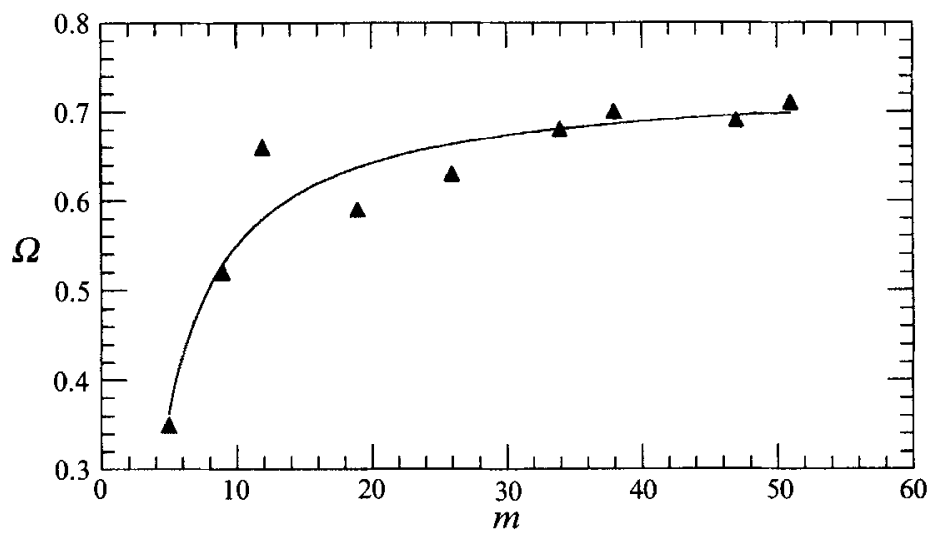

Figure 17. The angular velocity of $\Omega$, in units of deg. longitude per hour, versus the azimuthal wavenumber $m$. The data points correspond to the solid circles in figure 8 of Sromovsky et al. (1983) for wavenumbers $5,9,12,19,26,34,38,47$ and 51 . The line is our fit to the dispersion relation for interfacial Rossby waves.

$\begin{array}{crr}m & \Omega^{\prime}\left(\Omega_{e}\right) & T^{\prime} \text { (days) } \\ 1 & -1.000 & -1.0 \\ 2 & -0.285 & -3.5 \\ 3 & -0.046 & -21.4 \\ 4 & 0.073 & 13.8 \\ 5 & 0.144 & 6.9 \\ 6 & 0.197 & 5.2 \\ 7 & 0.226 & 4.4\end{array}$

TABLE 2. The angular velocities $\Omega^{\prime}=\Omega-\Omega_{e}$ (in units of the Earth's angular velocity $\Omega_{e}$ ) and the periods $T^{\prime}=2 \pi / \Omega^{\prime}$ (in days) for the linear waves propagating on the edge of a polar cap of uniform vorticity $2.6 \Omega_{e}$ extending from the pole to $55^{\circ}$, as a function of the azimuthal wavenumber $m$. The values are computed in a frame of reference that rotates with angular velocity $\Omega_{e}$, as they would be measured by an observer on the Earth.

function of their azimuthal wavenumber $m$. They have also showed how the Rossby wave dispersion relation is inadequate to fit the data because the angular velocity of Rossby waves decays too rapidly for small $m$ (it varies as $m^{-2}$ ). The dispersion relation (9) for interfacial Rossby waves decays only as $m^{-1}$, and therefore seems a better candidate to describe the waves on the ribbon.

We have fitted the angular velocity values obtained by Sromovsky et al. (1983) to the form $\Omega=\Omega_{0}-\tilde{\omega} /(2 m)$. The value of $\Omega_{0}$ is simply the angular velocity associated with the jet located at the vorticity interface; the fit gives $\Omega_{0}=0.74 \pm 0.02^{\circ}$ longitude/hour, corresponding to a jet peak of approximately $145 \mathrm{~ms}^{-1}$. The fit also provides the value of the vorticity jump across the ribbon; we find $\tilde{\omega}=3.72 \pm 0.45^{\circ}$ longitude/hour. As one may see from figure 17 , this good fit strongly suggests that the dynamics of waves on Saturn's ribbon is dictated by the steepness of the vorticity gradient over a very narrow latitudinal region; this explains why traditional Rossby wave dynamics, which rests on vorticity gradients that are evenly spread over the spherical surface, is unable to reproduce the observations, as Sromovsky et al. (1983) have shown.

D.G.D. is supported by a grant from the UK Science and Engineering Research 
Council. L. M.P. acknowledges support from the Atmospheric Science Division of the US National Science Foundation, and is grateful to M. E. McIntyre and his research group for their hospitality during his visits to Cambridge; he also wishes to thank R. A. Plumb, D. Waugh, T. Dowling, M. Allison and L. Sromovsky for several helpful discussions. The authors are also grateful to Vim Verkley for suggesting a hint on how to derive the last expression in Appendix A, and to R. Saravanan for helping with some of the graphics. Most the computations were carried out on the Crays YMP at the Rutherford Appleton Laboratory and the Pittsburgh Supercomputing Center; the latter is supported by the US National Science Foundation.

\section{Appendix A}

The constants $C$ and $\Omega^{\prime}$ in (14) are chosen so that the area enclosed by the vortex is equal to $A_{0}=2 \pi\left(1-z_{0}\right)$, and its aspect ratio is $\lambda$. Given the boundary shape $x(s)$, the area $A(x)$ of the polar cap is given by the expression

$$
A(\boldsymbol{x})=\oint \mathscr{Z} \mathrm{d} \varphi=\oint(1-z) \frac{x \mathrm{~d} y-y \mathrm{~d} x}{r^{2}}=\oint \frac{x y_{s}-y x_{s}}{1+z} \mathrm{~d} s .
$$

At the $n$th iteration, we must require that $A\left(x_{n+1}\right)=A_{0}$. Substituting (10) into the last equality above and using (13) yields, after some algebra, the equation $a_{1} C+b_{1} \Omega^{\prime}=$ $c_{1}$, where

$$
a_{1}=\oint \chi^{-1}\left(\boldsymbol{x}_{n}\right) \mathrm{d} s, \quad b_{1}=\oint \frac{\mathscr{Z}_{n}}{\chi\left(\boldsymbol{x}_{n}\right)} \mathrm{d} s, \quad c_{1}=A_{0}-A\left(\boldsymbol{x}_{n}\right)+\oint \frac{\psi_{r}\left(\boldsymbol{x}_{n}\right)}{\chi\left(\boldsymbol{x}_{n}\right)} \mathrm{d} s_{n} .
$$

In practice, owing to the symmetry of the problem, the integrals only need be evaluated over the interval $(0, \pi / m)$ (see figure 2 ).

The aspect-ratio constraint is imposed by requiring that

$$
\frac{\mathscr{Z}_{n+1}(\varphi=0)}{\mathscr{Z}_{n+1}(\varphi=\pi / m)}=\lambda
$$

Substituting (10) in this expression and using (13) yields the equation $a_{2} c+b_{2} \Omega^{\prime}=c_{2}$, where

$$
\begin{aligned}
& a_{2}=\lambda \frac{n_{z}(\pi / m)}{\chi(\pi / m)}-\frac{n_{z}(0)}{\chi(0)}, \quad b_{2}=\lambda \frac{n_{z}(\pi / m) \mathscr{Z}(\pi / m)}{\chi(\pi / m)}-\frac{n_{z}(0) \mathscr{Z}(0)}{\chi(0)}, \\
& c_{1}=\lambda\left(\mathscr{Z}(\pi / m)+\frac{n_{z}(\pi / m) \psi_{r}(\pi / m)}{\chi(\pi / m)}\right)-\left(\mathscr{Z}(0)+\frac{n_{z}(0) \psi_{r}(0)}{\chi(0)}\right) .
\end{aligned}
$$

Finally, expressions are needed to evaluate $\psi$ and $\chi$ in terms of contour integrals. Some elementary but convoluted algebraic manipulations yield

$$
\chi(\boldsymbol{x})=\frac{1}{1-z^{2}}\left(w z_{s}-n_{z} \frac{\partial \psi}{\partial \mathscr{Z}}\right)+\Omega n_{z} .
$$

As for $\psi$ itself, we have derived, after much algebra, the following simple expression:

$$
\psi(x)=-\frac{1}{\pi} \sum_{k} \tilde{\omega}_{k} \oint_{\mathscr{C}_{k}} \frac{\log \left(\frac{1}{2}\left|x-X_{k}\right|\right)}{4-\left|x-X_{k}\right|^{2}} \mathrm{~d} X_{k} .
$$




\section{Appendix B}

The general scheme for computing multi-vortex equilibria is very similar to the one described in $\S 2.3$ and Appendix A. The area constraint yields, as for the wave problem, an equation of the form $a_{1} C+b_{1} \Omega^{\prime}=c_{1}$. The constants are the same as the ones given in Appendix $\mathrm{A}$, with the proviso that the integrals are taken around a single vortex, and that the area $A_{0}$ of each vortex is given by $A_{0} \equiv=(4 \pi \mu) / N$ (recall that $\mu$ is the fractional area of the total array of vortices).

The second equation for $C$ and $\Omega^{\prime}$ is obtained by enforcing that the centroid of vorticity $X$ of each vortex be held fixed during the iteration. Letting

$$
\boldsymbol{J} \equiv \oint \boldsymbol{x}_{n} \times \mathrm{d} \boldsymbol{x}_{n}
$$

where $\boldsymbol{x}_{n}$ is the position of the vortex boundary after the $n$th iteration, the expression for the centroid of each vortex is simply $X=J /|J| \equiv(X, Y, Z)$. Since by construction (and without loss of generality) we set $Y=0$, and since the centroid lies on a sphere so that $X=\left(1-Z^{2}\right)^{\frac{1}{2}}$, the constraint reduces to imposing that $Z=\sin \vartheta_{0}=z_{0}$. This leads to a linear equation of the form

$$
a_{2} C+b_{2} \Omega^{\prime}=c_{2},
$$

where $a_{2}, b_{2}$ and $c_{2}$ are given by the following expressions, obtained after substitution of (10) into the above definition for $Z$, and linearization:

$$
a_{2}=\oint \frac{\xi\left(\boldsymbol{x}_{n}\right)}{\chi\left(\boldsymbol{x}_{n}\right)} \mathrm{d} s, \quad b_{2}=-\oint \frac{\xi\left(\boldsymbol{x}_{n}\right) z_{n}}{\chi\left(\boldsymbol{x}_{n}\right)} \mathrm{d} s, \quad c_{2}=\oint \frac{\xi\left(\boldsymbol{x}_{n}\right) \psi_{r}\left(\boldsymbol{x}_{n}\right)}{\chi\left(\boldsymbol{x}_{n}\right)} \mathrm{d} s+\frac{z_{0}-Z}{2 X},
$$

with $\xi(x) \equiv=X z-Z x$.

\section{REFERENCES}

Bowman, K. P. 1992 Observations of deformation and mixing of the total ozone field in the antarctic polar vortex. J. Atmos. Sci. (in press).

BusSE, F. H. 1975 Patterns of convection in spherical shells. J. Fluid Mech. 72, 67-85.

DeEM, G. S. \& ZABUSKY, N. J. 1978 Stationary V-states: interactions, recurrence and breaking. Phys. Re?. Lett. 40, 859.

DhanaK, M. R. 1992 Stability of a regular polygon of finite vortices. J. Fluid Mech. 234, 297-316.

Dritschel, D. G. 1985 The stability and energetics of co-rotating uniform vortices. J. Fluid Mech. $157,95-134$.

Dritschel, D. G. 1988 a Nonlinear stability bounds for inviscid, two-dimensional, parallel or circular flows with monotonic vorticity, and the analogous three-dimensional quasi-geostrophic flows. J. Fluid Mech. 191, 575-582.

DritsChel, D. G. $1988 b$ The repeated filamentation of two-dimensional vorticity interfaces. $J$. Fluid Mech. 194, 511-532.

Dritschel, D. G. 1988 c Contour dynamics/surgery on the sphere. J. Comput. Phys. 79, 477-483.

DRITSCHEL, D. G. 1989 Contour dynamics and contour surgery: numerical algorithms for extended, high-resolution modelling of vortex dynamics in two-dimensional, inviscid, incompressible flows. Comput. Phys. Rep. 10, 77-146.

Dritschel, D. G. \& Legras, B. 1993 Vortex stripping. J. Fluid Mech. (submitted).

Dritschel, D. G. \& MCINTYRe, M. E. 1990 Does contour dynamics go singular? Phys. Fluids A 2, 748-753.

Dritschel, D. G. \& Polvani, L. M. 1992 The roll-up of vorticity strips on the surface of a sphere. J. Fluid Mech. 234, 47-69.

Ingersoll, A. P., Beebe, R. E., Conrath, B. J. \& Hunt, G. E. 1984 Structure and dynamics of Saturn's atmosphere. In Saturn (ed. T. Gehrels \& M. S. Matthews). University of Arizona Press. 
JuCKES, M. N. \& MCINTYRE, M. E. 1987 A high-resolution one-layer model of braking planetary waves in the stratosphere. Nature 328, 590-596.

Kimura, Y. \& OKamoto, H. 1987 Vortex motion on a sphere. J. Phys. Soc. Japan 56, 4203-4206.

LAMB, H. H. 1932 Hydrodynamics. Dover.

MCINTYRE, M. E. 1991 Atmospheric dynamics: some fundamentals with observational implications. In Proc. Intl School of Physics 'Enrico Fermi', CXV Course (ed. J. C. Gille \& G. Visconti).

Pierrehumbert, R. T. 1980 A family of steady translating vortex pairs with distributed vorticity. J. Fluid Mech. 99, 129-144.

Polvani, L. M. 1991 Two-layer geostrophic vortex dynamics. Part 2. Alignment an two-layer Vstates. J. Fluid Mech. 225, 241-270.

Polvani, L. M. \& CaRTon, X. J. 1990 The tripole a new coherent vortex structure of incompressible two-dimensional flows. Geophys. Astrophys. Fluid Dyn. 52, 87-102.

Polvani, L. M., Flierl, G. R. \& ZabusKY, N. J. $1989 a$ Filamentation of coherent vortex structures via separatrix crossing: a quantitative estimate of onset time. Phys. Fluids A 2, 181-184.

Polvani, L. M. \& Plumb, R. A. 1993 Rossby wave breaking, microbreaking, filamentation and secondary vortex formation: the dynamics of a perturbed vortex. J. Atmos. Science (in press).

Polvani, L. M., Zabusky, N. J. \& Flierl, G. R. $1989 b$ Two-layer geostrophic vortex dynamics. Part 1. Upper-layer V-states and merger. J. Fluid Mech. 205, 215-242.

SAFFMAN, P. G. \& SchatzMan, J. C. 1982 Stability of vortex street of finite vortices. J. Fluid Mech. $117,171-185$.

Saffman, P. G. \& Szeto, R. 1980 Equilibrium shapes for a pair of uniform vortices. Phys. Fluids 23, 2339-2342.

Smith, B. A. et al. 1982 A new look at the Saturn system: the Voyager 2 images. Science 215, 504-537.

Sromovsky, L. A., Revercomb, H. E., Krauss, R. J. \& Soumi, V. E. 1983 Voyager 2 observations of Saturn's northern mid-latitude cloud features: morphology, motions and evolution. J. Geophys. Res. 88, 8650-8666.

Thomson, J. J. 1883 A Treatise on the Motion of Vortex Rings, pp. 94-108. Macmillan.

WaUgh, D. W. 1992 Contour surgery simulations of a forced polar vortex. J. Atmos. Sci. 50, 714-730.

ZABUSKY, N. J. 1981 Recent developments in contour dynamics for the Euler equations in two dimensions. Ann. N.Y. Acad. Sci. 373, 160-170. 